\title{
ON LEAST-SQUARES FINITE ELEMENT METHODS FOR THE POISSON EQUATION AND THEIR CONNECTION TO THE DIRICHLET AND KELVIN PRINCIPLES*
}

\author{
PAVEL BOCHEV ${ }^{\dagger}$ AND MAX GUNZBURGER ${ }^{\ddagger}$
}

\begin{abstract}
Least-squares finite element methods for first-order formulations of the Poisson equation are not subject to the inf-sup condition and lead to stable solutions even when all variables are approximated by equal-order continuous finite element spaces. For such elements, one can also prove optimal convergence in the "energy" norm (equivalent to a norm on $H^{1}(\Omega) \times H(\Omega$, div)) for all variables and optimal $L^{2}$ convergence for the scalar variable. However, showing optimal $L^{2}$ convergence for the flux has proven to be impossible without adding the redundant curl equation to the first-order system of partial differential equations. In fact, numerical evidence strongly suggests that nodal continuous flux approximations do not posses optimal $L^{2}$ accuracy. In this paper, we show that optimal $L^{2}$ error rates for the flux can be achieved without the curl constraint, provided that one uses the div-conforming family of Brezzi-Douglas-Marini or Brezzi-Douglas-Duran-Fortin elements. Then, we proceed to reveal an interesting connection between a least-squares finite element method involving $H(\Omega$, div)-conforming flux approximations and mixed finite element methods based on the classical Dirichlet and Kelvin principles. We show that such least-squares finite element methods can be obtained by approximating, through an $L^{2}$ projection, the Hodge operator that connects the Kelvin and Dirichlet principles. Our principal conclusion is that when implemented in this way, a least-squares finite element method combines the best computational properties of finite element methods based on each of the classical principles.
\end{abstract}

Key words. least-squares finite element methods, Kelvin principle, Dirichlet principle, BDM spaces, BDDF spaces, RT spaces, mixed methods, Poisson equation

AMS subject classifications. 76D05, 76D07,65F10,65F 30

DOI. $10.1137 /$ S003614290443353X

1. Introduction. Stable mixed finite element methods for the Poisson equation ${ }^{1}$ (written in a first-order form in terms of a scalar variable and a flux) require the use of finite element spaces that satisfy an appropriate inf-sup condition. For methods based on the Dirichlet principle, the inf-sup condition can be easily satisfied but for the dual Kelvin principle, it imposes complicated restrictions on the choice of spaces; see [11]. In either case, it is well known that pairs of standard nodal-based, continuous finite element spaces fail the inf-sup condition and lead to unstable mixed methods. It is also well known that the inf-sup condition is circumvented if one uses such simple element pairs in finite element methods based on $L^{2}$ least-squares variational principles. Ever since such least-squares finite element methods for firstorder formulations of the Poisson equation were first considered in [24], this fact

${ }^{*}$ Received by the editors August 25, 2003; accepted for publication September 3, 2004; published electronically June 14, 2005. This work was funded by the Applied Mathematical Sciences program, U.S. Department of Energy, Office of Energy Research, and performed at Sandia National Labs, a multiprogram laboratory operated by Sandia Corporation, a Lockheed-Martin Company, for the United States Department of Energy's National Nuclear Security Administration under contract DEAC-94AL85000.

http://www.siam.org/journals/sinum/43-1/43353.html

${ }^{\dagger}$ Computational Mathematics and Algorithms Department, Sandia National Laboratories, Albuquerque, NM 87185-1110 (pbboche@sandia.gov).

${ }^{\ddagger}$ School of Computational Science, Florida State University, Tallahassee, FL 32306-4120 (gunzburg@csit.fsu.edu). The research of this author was supported in part by CSRI, Sandia National Laboratories, under contract 18407.

${ }^{1}$ Although we consider only the Poisson problem, much of what we discuss can be easily extended to more general second-order elliptic partial differential equations. 
has been deemed as an important advantage of those methods over mixed Galerkin methods.

Already in [24], optimal $L^{2}$ error estimates for least-square finite element methods were established for the scalar variable; however, there, no optimal $L^{2}$ convergence results were obtained for nodal approximations of the flux. This situation persisted in all subsequent analyses: optimal $L^{2}$ error estimates for the flux could not be obtained ${ }^{2}$ without the addition of a "redundant" curl equation; see, e.g., [13, 14, 15, 25, 27]. Moreover, computational studies in [16] strongly suggested that optimal $L^{2}$ convergence may in fact be nearly impossible if one uses pairs of standard nodal-based continuous finite element spaces. A notable exception was a case studied in [16] for which optimal $L^{2}$ error estimates for both the scalar variable and the flux were obtained when these variables were approximated by continuous nodal spaces built on a criss-cross grid. The key to their proof was the validity of the grid decomposition property (GDP) which was established for the criss-cross grid in [17]. So far, the criss-cross grid remains the only known case of a continuous nodal-based finite element space for which the GDP can be verified. More importantly, it was shown in [17] (see also [7]) that the GDP is necessary and sufficient for the stability of the mixed finite element method based on the Kelvin principle.

The correlation between the stability of mixed finite element methods and the optimal accuracy of least-squares finite element methods, established in [16], opens up the intriguing possibility that optimal $L^{2}$ accuracy for the flux may be obtainable for a least-squares finite element method, provided that this variable is approximated by $H(\Omega$, div)-conforming elements that are stable for mixed finite element methods based on the Kelvin principle. Today, the stability of mixed finite element methods based on the Kelvin principle is well understood, and many examples of stable finite element pairs are known. The first goal of our study is to show that the use of some of these spaces in a least-squares finite element method will indeed help to improve the $L^{2}$ accuracy of the flux approximation. Our second goal is to offer a new perspective on least-squares principles as resulting from a choice for the approximation of the Hodge *-operator that connects two mutually dual "energy" principles. Among other things, such an interpretation shows, in our context, why the use of $H(\Omega$, div)-conforming elements is in fact more natural than the use of equal-order $C^{0}$ spaces.

While our conclusions may disappoint the adherents of equal-order implementations, our results do not void least-squares finite element methods as a viable computational alternative. To the contrary, they demonstrate that when implemented correctly, a least-squares finite element method combines the best computational properties of finite element methods based on both the Dirichlet and Kelvin principles, and at the same time manages to avoid the indefinite linear systems that make the latter more difficult to solve. Although we reach this conclusion in the specific context of mixed and least-squares finite element methods for the Poisson problem, the idea of defining the latter type of method so that it inherits the best characteristics of a pair of mixed methods that are related through duality may have considerably wider application.

In the rest of this section, we briefly review the notation used throughout the paper. Then, in section 2.1, we recall the Dirichlet and Kelvin principles and the

\footnotetext{
${ }^{2}$ A somewhat different situation exists for negative-norm-based least-squares finite element methods, for which it is known that the $L^{2}$ accuracy of the flux is optimal with respect to the spaces used; however, for such methods, no error bound for the divergence of the flux could be established; see $[10]$.
} 
associated first-order div-grad formulation of the boundary value problem for the Poisson equation. There, in the context of the Kelvin principle, we also review basic definitions and properties of stable $H(\Omega$, div)-conforming mixed finite elements spaces for the flux and show that they satisfy the GDP. For the sake of brevity, we restrict attention to the well-known Raviart-Thomas (RT), Brezzi-Douglas-Marini (BDM), and Brezzi-Douglas-Duran-Fortin (BDDF) classes of affine families of finite elements. Section 3 deals with least-squares finite element methods for first-order formulations of the Poisson problem. After a brief review of known error estimates in $H^{1}(\Omega) \times$ $H\left(\Omega\right.$, div), we turn our attention to the $L^{2}$ accuracy and the rarely discussed case of least-squares finite element methods using RT, BDM, or BDDF approximations of the flux. We show that BDM and BDDF spaces lead to optimal convergence of the flux in $L^{2}$. In section 4 , we offer an interpretation of such least-squares finite element methods which is derived with the help of some notions from exterior calculus and differential forms.

1.1. Notation. Throughout, $\Omega$ denotes a bounded region in $\mathbb{R}^{n}, n=2,3$, with a Lipschitz continuous boundary $\Gamma=\partial \Omega$. We assume that $\Gamma$ consists of two disjoint parts denoted by $\Gamma_{D}$ and $\Gamma_{N}$. For $p>0, H^{p}(\Omega)$ denotes the Sobolev space of order $p$ with norm and inner product denoted by $\|\cdot\|_{p}$ and $(\cdot, \cdot)_{p}$, respectively. When $p=0$, we use the standard notation $L^{2}(\Omega)$. The symbol $|\cdot|_{k}, 0 \leq k \leq p$, denotes the $k$ th seminorm on $H^{p}(\Omega)$. Vector-valued functions and vector analogues of the Sobolev spaces are denoted by lower- and upper case bold-face font, respectively, e.g., $\mathbf{u}, \mathbf{H}^{1}(\Omega), \mathbf{L}^{2}(\Omega)$, etc. We recall the space

$$
H(\Omega, \operatorname{div})=\left\{\mathbf{u} \in \mathbf{L}^{2}(\Omega) \mid \nabla \cdot \mathbf{u} \in L^{2}(\Omega)\right\},
$$

which is a Hilbert space when equipped with the norm

$$
\|\mathbf{u}\|_{H(\Omega, \operatorname{div})}=\left(\|\mathbf{u}\|_{0}^{2}+\|\nabla \cdot \mathbf{u}\|_{0}^{2}\right)^{1 / 2}
$$

To deal with the boundary conditions, we introduce the spaces

$$
H_{D}^{1}(\Omega)=\left\{\phi \in H^{1}(\Omega) \mid \phi=0 \quad \text { on } \Gamma_{D}\right\}
$$

and

$$
H_{N}(\Omega, \operatorname{div})=\left\{\mathbf{v} \in H(\Omega, \operatorname{div}) \mid \mathbf{v} \cdot \mathbf{n}=0 \quad \text { on } \Gamma_{N}\right\} .
$$

Details about all the notation just introduced may be found, e.g., in [11, 18].

Throughout, we will refer to the problem

$$
-\Delta \phi+\gamma \phi=f \quad \text { in } \Omega, \quad \phi=0 \quad \text { on } \Gamma_{D}, \quad \text { and } \quad \partial \phi / \partial n=0 \quad \text { on } \Gamma_{N}
$$

as the Poisson problem, even though that terminology is usually reserved for the case $\gamma=0$.

2. Mixed finite element methods for the Poisson problem. So as to provide a background for some of the discussions of sections 3 and 4 concerning leastsquares finite element methods, we consider, in this section, primal and dual mixed finite element methods for the Poisson problem.

2.1. The generalized Dirichlet and Kelvin principles. The Dirichlet and Kelvin principles arise in a variety of applications. Mathematically, they provide two variational formulations for the Poisson problem and also form the basis for defining mixed finite element methods for approximations of the solution of that problem. 
2.1.1. The generalized Dirichlet principle. Consider the functional

$$
D(\phi, \mathbf{w} ; f)=\frac{1}{2} \int_{\Omega}\left(|\mathbf{w}|^{2}+\gamma|\phi|^{2}\right) d \Omega-\int_{\Omega} f \phi d \Omega
$$

and the minimization problem

$$
\min _{(\phi, \mathbf{w}) \in H_{D}^{1}(\Omega) \times \nabla H_{D}^{1}(\Omega)} D(\phi, \mathbf{w} ; f) \quad \text { subject to } \quad \mathbf{w}+\nabla \phi=0,
$$

where $\gamma \geq 0$ is a given function that is assumed to satisfy $\|\gamma\|_{L^{\infty}(\Omega)} \leq C$ for some constant $C \geq 0$. The minimization principle (2.1) is known as the (generalized) Dirichlet principle. $^{3}$ Although the constraint $\mathbf{w}+\nabla \phi=0$ can be directly substituted into the functional to eliminate the flux $\mathbf{w},{ }^{4}$ it will be more profitable for our discussions to continue to consider the form (2.1).

With the help of a Lagrange multiplier $\mathbf{u}$ to enforce the constraint $\mathbf{w}+\nabla \phi=0$ and the Lagrangian functional

$$
L_{D}(\phi, \mathbf{w}, \mathbf{u} ; f)=\frac{1}{2} \int_{\Omega}\left(|\mathbf{w}|^{2}+\gamma|\phi|^{2}\right) d \Omega-\int_{\Omega} f \phi d \Omega-\int_{\Omega} \mathbf{u} \cdot(\mathbf{w}+\nabla \phi) d \Omega,
$$

the constrained minimization problem (2.1) can be transformed into the unconstrained optimization problem of determining saddle-points $(\phi, \mathbf{w}, \mathbf{u}) \in H_{D}^{1}(\Omega) \times \nabla H_{D}^{1}(\Omega) \times$ $\nabla H_{D}^{1}(\Omega)$ of $L_{D}(\phi, \mathbf{w}, \mathbf{u} ; f)$. It is not difficult to see that the optimality system obtained by setting the first variations of $L_{D}(\phi, \mathbf{w}, \mathbf{u} ; f)$ to zero is given by the following: seek $(\phi, \mathbf{w}, \mathbf{u}) \in H_{D}^{1}(\Omega) \times \nabla H_{D}^{1}(\Omega) \times \nabla H_{D}^{1}(\Omega)$ such that

$$
\left\{\begin{aligned}
\int_{\Omega} \mathbf{w} \cdot \mathbf{v} d \Omega+\int_{\Omega} \nabla \phi \cdot \mathbf{v} d \Omega & =0 & & \forall \mathbf{v} \in \nabla H_{D}^{1}(\Omega), \\
\int_{\Omega}(\mathbf{w}-\mathbf{u}) \cdot \mathbf{q} d \Omega & =0 & & \forall \mathbf{q} \in \nabla H_{D}^{1}(\Omega), \\
-\int_{\Omega} \mathbf{u} \cdot \nabla \psi d \Omega+\int_{\Omega} \gamma \phi \psi d \Omega & =\int_{\Omega} f \psi d \Omega & & \forall \psi \in H_{D}^{1}(\Omega) .
\end{aligned}\right.
$$

The first and second equations may be easily combined to yield the simplified system

$$
\begin{cases}\int_{\Omega} \mathbf{u} \cdot \mathbf{v} d \Omega+\int_{\Omega} \nabla \phi \cdot \mathbf{v} d \Omega=0 & \forall \mathbf{v} \in \nabla H_{D}^{1}(\Omega), \\ \int_{\Omega} \nabla \psi \cdot \mathbf{u} d \Omega-\int_{\Omega} \gamma \psi \phi d \Omega=-\int_{\Omega} f \psi d \Omega & \forall \psi \in H_{D}^{1}(\Omega),\end{cases}
$$

involving only $\phi \in H_{D}^{1}(\Omega)$ and $\mathbf{u} \in \nabla H_{D}^{1}(\Omega)$.

If solutions to the constrained minimization problem (2.1) or, equivalently, of (2.4), are sufficiently smooth, then without much difficulty one obtains that

\footnotetext{
${ }^{3}$ For $f=0, \gamma=0$, and appropriate boundary conditions, the Dirichlet principle in the inviscid fluid mechanics setting states that among all irrotational velocity fields, the one that minimizes the kinetic energy is the solenoidal one. In the solid mechanics setting, w is a tensor, and a simplified version of (2.1) is the energy minimization principle.

${ }^{4}$ This results in the certainly more familiar form for the (generalized) Dirichlet principle:

$$
\min _{\phi \in H_{D}^{1}(\Omega)} \widetilde{D}(\phi ; f), \quad \text { where } \quad \widetilde{D}(\phi ; f)=\frac{1}{2} \int_{\Omega}\left(|\nabla \phi|^{2}+\gamma|\phi|^{2}\right) d \Omega-\int_{\Omega} f \psi d \Omega .
$$
}




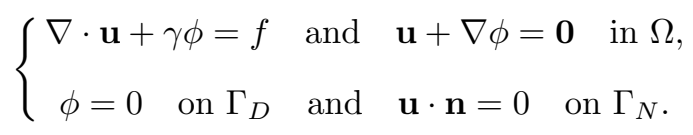

Note that from the second equation of $(2.3)$ we also have that $\mathbf{w}=\mathbf{u}$. Eliminating the flux $\mathbf{u}$ from (2.5) (again assuming that sufficient smoothness is available), one obtains the Poisson problem ${ }^{5}$ (1.1) for the scalar variable $\phi$.

2.1.2. The generalized Kelvin principle. Now consider the functional

$$
K(\lambda, \mathbf{u})=\frac{1}{2} \int_{\Omega}\left(|\mathbf{u}|^{2}+\gamma|\lambda|^{2}\right) d \Omega
$$

and the minimization problem

$$
\min _{\lambda \in L^{2}(\Omega), \mathbf{u} \in H_{N}(\Omega, \text { div })} K(\lambda, \mathbf{v}) \quad \text { subject to } \nabla \cdot \mathbf{u}+\gamma \lambda=f .
$$

The minimization principle (2.7) is known as the (generalized) Kelvin principle ${ }^{6}$ it is dual to the (generalized) Dirichlet principle. ${ }^{7}$

With the help of a Lagrange multiplier $\phi$ to enforce the constraint and the Lagrangian functional

$$
L_{K}(\lambda, \mathbf{u}, \phi ; f)=\frac{1}{2} \int_{\Omega}\left(|\mathbf{u}|^{2}+\gamma|\lambda|^{2}\right) d \Omega-\int_{\Omega} \phi(\nabla \cdot \mathbf{u}+\gamma \lambda-f) d \Omega,
$$

the constrained minimization problem $(2.7)$ can be transformed into the unconstrained problem of determining saddle-points $(\lambda, \mathbf{u}, \phi) \in L^{2}(\Omega) \times H_{N}(\Omega$, div $) \times L^{2}(\Omega)$ of $L_{K}(\lambda, \mathbf{u}, \phi ; f)$. It is not difficult to see that the optimality system obtained by setting

\footnotetext{
${ }^{5}$ Note that since $\nabla \psi \in \mathbf{L}^{2}(\Omega)$, one can easily combine the two equations in (2.4) to yield the more familiar weak formulation

$$
\int_{\Omega} \nabla \phi \cdot \nabla \psi d \Omega+\int_{\Omega} \gamma \psi \phi d \Omega=\int_{\Omega} f \psi d \Omega \quad \forall \psi \in H_{D}^{1}(\Omega)
$$

for the Poisson problem (1.1). Again, it will be more profitable for our discussion to continue to use (2.4) instead of the more familiar form (2.6).

${ }^{6}$ Setting $f=0$ and $\gamma=0$ and allowing for an inhomogeneous boundary condition for $\mathbf{u} \cdot \mathbf{n}$, the Kelvin principle for inviscid flows states that, among all incompressible velocity fields, the one that minimizes the kinetic energy is irrotational. In structural mechanics (where $\mathbf{u}$ is a tensor), a simplified version of (2.7) is known as the complimentary energy principle.

${ }^{7}$ Unlike the case of the Dirichlet principle, if $\gamma=0$, one cannot directly use the constraint $\nabla \cdot \mathbf{u}+\gamma \lambda=f$ to eliminate one of the variables. If $\gamma>0$, then it is possible to use the constraint to eliminate the scalar variable $\lambda$. In fact, in the latter case we are led to the problem

$$
\min _{\mathbf{u} \in H_{N}(\Omega, \operatorname{div})} \widetilde{K}(\mathbf{u} ; f), \quad \text { where } \quad \widetilde{K}(\mathbf{u} ; f)=\frac{1}{2} \int_{\Omega}\left(|\mathbf{u}|^{2}+\frac{1}{\gamma}|\nabla \cdot \mathbf{u}-f|^{2}\right) d \Omega .
$$

Comparing (2.2) and (2.8), we already see a big difference between the Kelvin and Dirichlet principles, in addition to the obvious difficulty seen in $(2.8)$ for the case $\gamma=0$. The functional $\widetilde{D}(\cdot ; f)$ in $(2.2)$ involves all first derivatives of the scalar variable $\phi$, which is why we can minimize it over the space $H_{D}^{1}(\Omega)$. On the other hand, the functional $\widetilde{K}(\cdot ; f)$ in $(2.8)$ only involves the combination $\nabla \cdot \mathbf{u}$ of first derivatives of the flux $\mathbf{u}$, which is why we can minimize it only with respect to a subspace of $H(\Omega, \operatorname{div})$, and not with respect to $\mathbf{H}^{1}(\Omega)$.
} 
the first variations of $L(\lambda, \mathbf{u}, \phi ; f)$ to zero is given by the following: seek $(\lambda, \mathbf{u}, \phi) \in$ $L^{2}(\Omega) \times H_{N}(\Omega, \operatorname{div}) \times L^{2}(\Omega)$ such that

$$
\left\{\begin{aligned}
\int_{\Omega} \mathbf{u} \cdot \mathbf{v} d \Omega-\int_{\Omega} \phi \nabla \cdot \mathbf{v} d \Omega & =0 & & \forall \mathbf{v} \in H_{N}(\Omega, \operatorname{div}), \\
\int_{\Omega} \gamma(\lambda-\phi) \mu d \Omega & =0 & & \forall \mu \in L^{2}(\Omega), \\
-\int_{\Omega} \psi \nabla \cdot \mathbf{u} d \Omega-\int_{\Omega} \gamma \psi \lambda d \Omega & =-\int_{\Omega} f \psi d \Omega & & \forall \psi \in L^{2}(\Omega) .
\end{aligned}\right.
$$

For $\gamma \neq 0$, the second and third equations may be easily combined to yield the simplified system

$$
\left\{\begin{aligned}
\int_{\Omega} \mathbf{u} \cdot \mathbf{v} d \Omega-\int_{\Omega} \phi \nabla \cdot \mathbf{v} d \Omega & =0 & & \forall \mathbf{v} \in H_{N}(\Omega, \operatorname{div}) \\
-\int_{\Omega} \psi \nabla \cdot \mathbf{u} d \Omega-\int_{\Omega} \gamma \psi \phi d \Omega & =-\int_{\Omega} f \psi d \Omega & & \forall \psi \in L^{2}(\Omega)
\end{aligned}\right.
$$

involving only $\phi \in L^{2}(\Omega)$ and $\mathbf{u} \in H_{N}(\Omega$, div $)$. If $\gamma=0$, then (2.9) directly reduces to (2.10) so that the latter holds for any $\gamma \geq 0$.

The duality of the Dirichlet and Kelvin principles extends to the optimality systems (2.4) and (2.10). For example, they are respectively described using the dual operators $\nabla$ and $-\nabla \cdot$. The domain of $\nabla$ is all of $H_{D}^{1}(\Omega)$, while its range is a constrained subspace of $\mathbf{L}^{2}(\Omega)$ consisting of irrotational functions. In contrast, the domain of $\nabla$. is a constrained subspace of $\mathbf{L}^{2}(\Omega)$ and its range is all of $L^{2}(\Omega)$. We note again the difference between the domain spaces of the two operators: $H_{D}^{1}(\Omega)$ involves all first derivatives of the scalar variable, while $H_{N}(\Omega$, div $)$ only involves a combination of first derivatives of the flux.

2.2. Stable mixed finite element spaces. Finite element approximations of the mixed problems (2.4) and (2.10) are not stable unless the spaces chosen to approximate $\phi$ and $\mathbf{u}$ satisfy the inf-sup condition. To keep our presentation reasonably short and devoid of unnecessary technical details, we focus on affine families of stable spaces defined on simplicial triangulations $\mathcal{T}_{h}$ of the domain $\Omega$ into elements $\mathcal{K}$. In two dimensions, $\mathcal{K}$ are triangles, and in three dimensions, they are tetrahedra. The symbol $P_{k}(\mathcal{K})$ denotes the space of all polynomials of degree less than or equal to $k$ defined on $\mathcal{K}$.

Nodal $C^{0}$ finite element spaces built from $m$ th degree polynomials, $m \geq 1$, are denoted by ${ }^{8} \mathcal{W}_{m}^{0}(\Omega)$. We recall that there exists an interpolation operator $\mathcal{I}_{0}$ into $\mathcal{W}_{m}^{0}(\Omega)$ such that for any $\phi \in H^{m+1}(\Omega)$,

$$
\left\|\phi-\mathcal{I}_{0} \phi\right\|_{0}+h\left\|\nabla\left(\phi-\mathcal{I}_{0} \phi\right)\right\|_{0} \leq C h^{m+1}|\phi|_{m+1} .
$$

\footnotetext{
${ }^{8}$ The reasoning leading to the choice of notation $\mathcal{W}_{k}^{i}$ for the finite element spaces we employ will become clear later.
} 
We denote by $\mathcal{W}_{m}^{1}(\Omega)$ the space $\nabla\left(\mathcal{W}_{m}^{0}(\Omega)\right) .{ }^{9}$ We will use the pair of finite element spaces $\mathcal{W}_{m}^{0}(\Omega)$ and $\mathcal{W}_{m}^{1}(\Omega)$ to discretize the Dirichlet principle.

For the Kelvin principle, we will use the ${ }^{10} \mathrm{BDM}_{k}$ and $\mathrm{RT}_{k}$ spaces on $\Omega$ that are built from the individual element spaces

$$
\operatorname{BDM}_{k}(\mathcal{K})=\left(P_{k}(\mathcal{K})\right)^{n} \text { and } \operatorname{RT}_{k}(\mathcal{K})=\left(P_{k}(\mathcal{K})\right)^{n}+\mathbf{x} P_{k}(\mathcal{K})
$$

in a manner that ensures the continuity of the normal component across element boundaries; see [11, pp. 113-116] for details and definitions of the corresponding element degrees of freedom. Since $\mathrm{BDM}_{k}$ and $\mathrm{RT}_{k}$ both contain complete polynomials of degree $k$, their approximation properties in $L^{2}$ are the same. In particular, one can show that for either the $\mathrm{BDM}_{k}$ or $\mathrm{RT}_{k}$ spaces there exists an interpolation operator $\mathcal{I}_{2}$ such that

$$
\left\|\mathbf{u}-\mathcal{I}_{2} \mathbf{u}\right\|_{0} \leq C h^{r}|\mathbf{u}|_{r} \quad \forall \mathbf{u} \in \mathbf{H}^{r}(\Omega) \text { and } 1 \leq r \leq k+1 .
$$

Since $\mathrm{RT}_{k}$ also contains the higher-degree polynomial component $\mathbf{x} P_{k}(\mathcal{K})$, it has better accuracy in $H\left(\Omega\right.$, div) than does $\mathrm{BDM}_{k}$. Note, however, that this additional component does not help to improve the $L^{2}$ accuracy of $\mathrm{RT}_{k}$ spaces because it does not increase the order of complete polynomials contained in $\mathrm{RT}_{k}$ to $k+1$. In summary, we have the following estimates for the error in the divergence of the interpolant (see $[11$, p. 132]):

$$
\left\|\nabla \cdot\left(\mathbf{u}-\mathcal{I}_{2} \mathbf{u}\right)\right\|_{0} \leq C h^{k}\|\nabla \cdot \mathbf{u}\|_{k} \quad \text { for } \mathrm{BDM}_{k} \text { spaces }
$$

and

$$
\left\|\nabla \cdot\left(\mathbf{u}-\mathcal{I}_{2} \mathbf{u}\right)\right\|_{0} \leq C h^{k+1}\|\nabla \cdot \mathbf{u}\|_{k+1} \quad \text { for } \mathrm{RT}_{k} \text { spaces. }
$$

In what follows, we will denote by $\mathcal{W}_{k}^{2}(\Omega)$ the $\mathrm{RT}$ and BDM spaces having equal approximation orders with respect to the divergence operator, i.e.,

$$
\mathcal{W}_{k}^{2}(\Omega)=\left\{\mathbf{v} \in H(\Omega, \operatorname{div})|\mathbf{v}|_{\mathcal{K}} \in \mathcal{W}_{k}^{2}(\mathcal{K})\right\},
$$

where $\mathcal{W}_{k}^{2}(\mathcal{K})$ is one of the finite element spaces $\mathrm{RT}_{k-1}(\mathcal{K})$ or $\operatorname{BDM}_{k}(\mathcal{K})$. We can now combine (2.13) and (2.14) into a single statement: there exists an interpolation operator $\mathcal{I}_{2}$ into $\mathcal{W}_{k}^{2}(\Omega)$ such that

$$
\left\|\nabla \cdot\left(\mathbf{u}-\mathcal{I}_{2} \mathbf{u}\right)\right\|_{0} \leq C h^{k}\|\nabla \cdot \mathbf{u}\|_{k} .
$$

Note, however, that from $(2.12)$ we have that the interpolation operator $\mathcal{I}_{2}$ into $\mathcal{W}_{k}^{2}(\Omega)$ satisfies

$$
\left\|\mathbf{u}-\mathcal{I}_{2} \mathbf{u}\right\|_{0} \leq C h^{r}|\mathbf{u}|_{r} \quad \begin{cases}\text { for } 1<r \leq k & \text { if } \mathcal{W}_{k}^{2}(\mathcal{K})=\mathrm{RT}_{k-1} \\ \text { for } 1<r \leq k+1 & \text { if } \mathcal{W}_{k}^{2}(\mathcal{K})=\mathrm{BDM}_{k}\end{cases}
$$

\footnotetext{
${ }^{9}$ In our setting, $\mathcal{W}_{m}^{1}(\Omega)$ is a space of vector-valued functions that are discontinuous with respect to the simplicial triangulation $\mathcal{T}_{h}$ and whose components belong to a subspace $P_{m-1}(\mathcal{K})$ in each $\mathcal{K}$. Functions belonging to $\mathcal{W}_{m}^{1}(\Omega)$ must be curl-free within each element $\mathcal{K}$ (since they are gradients of function belonging to $\mathcal{W}_{m}^{0}(\Omega)$ ), so that, except for $m=1$, they are not complete $(m-1)$ st degree polynomials. However, the precise, explicit characterization of $\mathcal{W}_{m}^{1}(\Omega)$, e.g., the construction of a basis, is not difficult (using their irrotational property), and moreover, as we shall see, it turns out not to be necessary in practice. For future reference, we note that functions belonging to the approximating space $\mathcal{W}_{m}^{0}(\Omega)$ for the scalar variable are continuous across element boundaries, so that the tangential components of functions belonging to the approximating space $\mathcal{W}_{m}^{1}(\Omega)=\nabla\left(\mathcal{W}_{m}^{0}(\Omega)\right)$ for the flux are automatically also continuous across element boundaries.

${ }^{10}$ To simplify notation, from now on we will denote both the BDM and BDDF spaces simply by BDM.
} 
We denote by $\mathcal{W}_{k}^{3}(\Omega)$ the space $\nabla \cdot\left(\mathcal{W}_{k}^{2}(\Omega)\right)$. For mixed finite element methods based on the Kelvin principle, we will use the finite element spaces $\mathrm{RT}_{k-1}$ or $\mathrm{BDM}_{k}$ to approximate the flux. For characterizations of these spaces and the associated spaces $\mathcal{W}_{k}^{3}(\Omega)=\nabla \cdot\left(\mathcal{W}_{k}^{2}(\Omega)\right)$ for the scalar variable, see $[11]$.

2.2.1. Stable mixed finite element spaces for the Dirichlet principle. A mixed finite element method based on the Dirichlet principle may be defined by discretizing (2.4), i.e.,

$$
\begin{cases}\int_{\Omega} \mathbf{u}_{h} \cdot \mathbf{v}_{h} d \Omega+\int_{\Omega} \nabla \phi_{h} \cdot \mathbf{v}_{h} d \Omega=0 & \forall \mathbf{v}_{h} \in \mathcal{W}_{m}^{1}(\Omega) \\ \int_{\Omega} \nabla \psi_{h} \cdot \mathbf{u}_{h} d \Omega-\int_{\Omega} \gamma \psi_{h} \phi_{h} d \Omega=-\int_{\Omega} f \psi_{h} d \Omega & \forall \psi \in \mathcal{W}_{m}^{0}(\Omega) .\end{cases}
$$

Since $\mathcal{W}_{m}^{1}(\Omega) \equiv \nabla\left(\mathcal{W}_{m}^{0}(\Omega)\right)$, note that, even at the discrete level, we may again eliminate the flux approximation to obtain the equivalent discrete problem

$$
\int_{\Omega} \nabla \phi_{h} \cdot \nabla \psi_{h} d \Omega+\int_{\Omega} \gamma \psi_{h} \phi_{h} d \Omega=\int_{\Omega} f \psi_{h} d \Omega \quad \forall \psi \in \mathcal{W}_{m}^{0}(\Omega)
$$

which we recognize as the standard Galerkin discretization of (2.2) or (2.6). In fact, using the pair of spaces $\mathcal{W}_{m}^{0}(\Omega)$ and $\mathcal{W}_{m}^{1}(\Omega)$ for approximating the scalar variable and the flux, respectively, in the discretization (2.17) of (2.4) is equivalent ${ }^{11}$ to using the scalar space $\mathcal{W}_{m}^{0}(\Omega)$ in the standard Galerkin discretization (2.18) of (2.2) and then letting the approximation of the flux be the gradient of the resulting approximation of the scalar variable.

In this way we see that for discretizations of (2.4), i.e., the Dirichlet principle, the required inf-sup condition is completely benign in the sense that it can be avoided by eliminating the flux approximation $\mathbf{u}_{h}$ from (2.17), then solving (2.18) for the approximation $\phi_{h}$ of the scalar variable using a standard continuous nodal finite element space $\mathcal{W}_{m}^{0}(\Omega)$, and, at the end, determining the approximation to the flux from the exact relation $\mathbf{u}_{h}=-\nabla \phi_{h}$. The required inf-sup condition is implicitly satisfied by the pair of spaces $\mathcal{W}_{m}^{0}(\Omega)$ and $\mathcal{W}_{m}^{1}(\Omega)=\nabla\left(\mathcal{W}_{m}^{0}(\Omega)\right)$. If one insists on solving (2.4), then one needs to explicitly produce a basis for $\mathcal{W}_{m}^{1}(\Omega)$; this is easily accomplished.

From either (2.17) or (2.18) one obtains, for the Dirichlet principle, that if $\phi \in$ $H^{m+1}(\Omega) \cap H_{D}^{1}(\Omega)$, then

$$
\left\|\phi-\phi_{h}\right\|_{0} \leq h^{m+1}\|\phi\|_{m+1},
$$

while the flux approximation is less accurate:

$$
\left\|\mathbf{u}-\mathbf{u}_{h}\right\|_{0}=\left\|\nabla\left(\phi-\phi_{h}\right)\right\|_{0} \leq h^{m}\|\phi\|_{m+1} .
$$

2.2.2. Stable mixed finite element spaces for the Kelvin principle. For discretizations of (2.10), i.e., the Kelvin principle, the inf-sup condition is much more onerous in the sense that defining a pair of stable finite element spaces for the scalar variable and the flux is not so straightforward a matter.

\footnotetext{
${ }^{11}$ Here, by equivalent we mean that they yield exactly the same approximate solutions.
} 
The mixed finite element method associated with (2.10), i.e., the Kelvin principle, is given by the following: seek $\left(\phi_{h}, \mathbf{u}_{h}\right) \in \mathcal{W}_{k}^{3}(\Omega) \times \mathcal{W}_{k}^{2}(\Omega)$ such that

$$
\begin{cases}\int_{\Omega} \mathbf{u}_{h} \cdot \mathbf{v}_{h} d \Omega-\int_{\Omega} \phi_{h} \nabla \cdot \mathbf{v}_{h} d \Omega=0 & \forall \mathbf{v}_{h} \in \mathcal{W}_{k}^{2}(\Omega), \\ \int_{\Omega} \psi_{h} \nabla \cdot \mathbf{u}_{h} d \Omega+\int_{\Omega} \gamma \psi_{h} \psi_{h} d \Omega=\int_{\Omega} f \psi_{h} d \Omega & \forall \psi_{h} \in \mathcal{W}_{k}^{3}(\Omega) .\end{cases}
$$

For the $\gamma=0$ case, we refer to [11] for a proof that $\left(\mathcal{W}_{k}^{3}(\Omega), \mathcal{W}_{k}^{2}(\Omega)\right)$ is a stable pair for the mixed finite element problem (2.21). Moreover, one can show [11, Proposition 1.2, p. 139] that for any sufficiently regular exact solution of (2.10) one has the error estimate

$$
\left\|\mathbf{u}-\mathbf{u}_{h}\right\|_{0} \leq C h^{r}\|\mathbf{u}\|_{r} \quad \begin{cases}\text { for } 1<r \leq k & \text { if } \mathcal{W}_{k}^{2}(\mathcal{K})=\mathrm{RT}_{k-1} \\ \text { for } 1<r \leq k+1 & \text { if } \mathcal{W}_{k}^{2}(\mathcal{K})=\mathrm{BDM}_{k}\end{cases}
$$

while the error in the divergence is of the same order in both cases,

$$
\left\|\nabla \cdot\left(\mathbf{u}-\mathbf{u}_{h}\right)\right\|_{0} \leq C h^{r}\|\nabla \cdot \mathbf{u}\|_{r} \quad \text { for } 1<r \leq k,
$$

as is the error in the scalar variable:

$$
\left\|\phi-\phi_{h}\right\|_{0} \leq C h^{r}\left(\|\phi\|_{r}+\|\mathbf{u}\|_{r}\right) \quad \text { for } 1<r \leq k .
$$

These results also hold for the $\gamma>0$ case, since the mixed finite element problem (2.21) is identical to what one obtains for penalty methods for the $\gamma=0$ case; see, e.g., $[11,18]$, for details.

We have thus seen that the duality between the Dirichlet and Kelvin principles propagates to their numerical approximations by mixed finite element methods that themselves have, in a sense, complementary computational properties. For example, for the Dirichlet principle, one directly approximates the scalar variable in the $H^{1}$ conforming finite element space $W_{m}^{0}(\Omega)$, and the flux is approximated in the finite element space $W_{m}^{1}(\Omega)=\nabla\left(W_{m}^{0}(\Omega)\right)$. With respect to $L^{2}(\Omega)$ norms, the mixed approximation $\phi_{h}$ to $\phi$ satisfies the optimal bound (2.19), while the approximation $\mathbf{u}_{h}$ of the flux $\mathbf{u}$ is less accurate; see (2.20). For the Kelvin principle, the situation is reversed in the sense that now one directly approximates the flux in the $H_{N}(\Omega$, div )-conforming finite element space $\mathcal{W}_{k}^{2}(\Omega)$ and the scalar variable in $\mathcal{W}_{k}^{3}(\Omega)=\nabla \cdot\left(\mathcal{W}_{k}^{2}(\Omega)\right)$. The approximation $\mathbf{u}_{h}$ to $\mathbf{u}$ now satisfies the optimal bound (2.22), while the scalar approximation is less accurate when $\mathcal{W}_{k}^{2}(\Omega)=\mathrm{BDM}_{k}$.

We have also seen the differences in how easily one can satisfy the inf-sup condition for mixed methods based on the two principles. From (2.18), one sees that for the Dirichlet principle one can essentially avoid the inf-sup condition, or, if one insists on using the mixed formulation (2.17), one can easily construct a stable pair of spaces. This is closely related to the fact that the null space of the gradient consists of the constant function and is trivial to approximate. On the other hand, for the Kelvin principle, one has to construct a pair of finite element spaces such that the space for approximating the scalar variable is the divergence of the space for approximating the flux and the latter is a subspace of $H(\Omega$, div). This is a much more difficult construction since the divergence operator has a decidedly nontrivial null space that is much harder to approximate than the (trivial) null space of the gradient. Compared to the finite element subspaces that can be used for approximations of the Dirichlet 
principle, for the Kelvin principle this leads to the need to define more complicated finite element subspaces for the flux such as the RT and BDM spaces or continuous piecewise linear subspaces based on the criss-cross grid.

It is important to note that if one uses $C^{0}$ finite element spaces for both the scalar variable and the flux, then (2.17) and (2.21) are identical discrete systems. It is well known that this leads to unstable approximations, so that one cannot use such pairs of finite element spaces in mixed methods derived from either the Dirichlet or Kelvin principles.

2.3. The grid decomposition property. We continue our study of mixed methods based on the Kelvin principle by showing that the spaces $\mathcal{W}_{k}^{2}(\Omega)$ satisfy the GDP. ${ }^{12}$

TheOREM 2.1. For every $\mathbf{u}_{h} \in \mathcal{W}_{k}^{2}(\Omega)$, there exist $\mathbf{w}_{h}, \mathbf{z}_{h}$ in $\mathcal{W}_{k}^{2}(\Omega)$ such that ${ }^{13}$

$$
\begin{gathered}
\mathbf{u}_{h}=\mathbf{w}_{h}+\mathbf{z}_{h}, \\
\nabla \cdot \mathbf{z}_{h}=0 \\
\int_{\Omega} \mathbf{w}_{h} \cdot \mathbf{z}_{h} d \Omega=0 \\
\left\|\mathbf{w}_{h}\right\|_{0} \leq C\left(\left\|\nabla \cdot \mathbf{u}_{h}\right\|_{-1}+h\left\|\nabla \cdot \mathbf{u}_{h}\right\|_{0}\right) .
\end{gathered}
$$

Proof. Given a $\mathbf{u}_{h} \in \mathcal{W}_{k}^{2}(\Omega)$, define $\mathbf{w}_{h}$ to be a solution of the following mixed problem: seek $\left(\phi_{h}, \mathbf{w}_{h}\right) \in \mathcal{W}_{k}^{3}(\Omega) \times \mathcal{W}_{k}^{2}(\Omega)$ such that

$$
\begin{array}{cc}
\int_{\Omega} \mathbf{w}_{h} \cdot \mathbf{v}_{h} d \Omega-\int_{\Omega} \phi_{h} \nabla \cdot \mathbf{v}_{h} d \Omega=0 & \forall \mathbf{v}_{h} \in \mathcal{W}_{k}^{2}(\Omega), \\
\int_{\Omega} \psi_{h} \nabla \cdot \mathbf{w}_{h} d \Omega=\int_{\Omega} \psi_{h} \nabla \cdot \mathbf{u}_{h} d \Omega & \forall \psi_{h} \in \mathcal{W}_{k}^{3}(\Omega) .
\end{array}
$$

The second component is then defined as the algebraic complement

$$
\mathbf{z}_{h}=\mathbf{u}_{h}-\mathbf{w}_{h}
$$

of $\mathbf{u}_{h}$. Therefore, the first GDP property (2.25) is trivially satisfied.

To prove (2.26), we use the second equation in (2.29) to conclude that

$$
\int_{\Omega} \psi_{h} \nabla \cdot \mathbf{z}_{h} d \Omega=\int_{\Omega} \psi_{h}\left(\nabla \cdot \mathbf{u}_{h}-\nabla \cdot \mathbf{w}_{h}\right) d \Omega=0 \quad \forall \psi_{h} \in \mathcal{W}_{k}^{3}(\Omega) .
$$

Assume now that $\nabla \cdot \mathbf{z}_{h} \neq 0$. From the definition of $\mathcal{W}_{k}^{3}(\Omega)$, it follows that the divergence operator is a surjective mapping $\mathcal{W}_{k}^{2}(\Omega) \mapsto \mathcal{W}_{k}^{3}(\Omega)$. Therefore, there exists

\footnotetext{
${ }^{12}$ An analogous "GDP" can be defined in the context of the Dirichlet principle; it requires that for every $\phi_{h} \in \mathcal{W}_{k}^{0}(\Omega)$ there exist $\lambda_{h}, \chi_{h} \in \mathcal{W}_{k}^{0}(\Omega)$ such that $\phi_{h}=\lambda_{h}+\chi_{h}, \nabla \chi_{h}=0, \int_{\Omega} \lambda_{h} \chi_{h} d \Omega=0$, and $\left\|\lambda_{h}\right\|_{0} \leq C\left(\left\|\nabla \phi_{h}\right\|_{-1}+h\left\|\nabla \phi_{h}\right\|_{0}\right)$. Of course, these conditions are trivially satisfied since $\nabla \chi_{h}=0$ and $\chi_{h} \in \mathcal{W}_{k}^{0}(\Omega)$ imply that $\chi_{h}=0$ and therefore $\lambda_{h}=\phi_{h}$. Again, the fact that the null space of the gradient operator with respect to $H_{D}^{1}(\Omega)$ is trivial plays a crucial role in the triviality of the GDP for the Dirichlet principle. On the other hand, for the Kelvin principle, the fact that the null space of the divergence operator with respect to $H_{N}(\Omega$, div $)$ is decidedly not trivial also plays a crucial role in the GDP for that principle. All this, of course, is related to the observations made above about the inf-sup conditions for the two principles.

${ }^{13}$ In its original form (see [17]), the GDP was formulated without the term $h\left\|\nabla \cdot \mathbf{u}_{h}\right\|_{0}$ in (2.28). However, thanks to the multiplicative $h$ factor, this term will not affect the $L^{2}$ error rates.
} 
a nonzero element $\widehat{\psi}_{h} \in \mathcal{W}_{k}^{3}(\Omega)$ such that $\widehat{\psi}_{h}=\nabla \cdot \mathbf{z}_{h}$. Then,

$$
0=\int_{\Omega} \widehat{\psi}_{h} \nabla \cdot \mathbf{z}_{h} d \Omega=\int_{\Omega} \widehat{\psi}_{h} \widehat{\psi}_{h} d \Omega \neq 0
$$

a contradiction.

To show that $\mathbf{w}_{h}$ and $\mathbf{z}_{h}$ are orthogonal, we use the first equation in (2.29) with $\mathbf{v}_{h}=\mathbf{z}_{h}$ :

$$
\int_{\Omega} \mathbf{w}_{h} \cdot \mathbf{z}_{h} d \Omega=\int_{\Omega} \phi_{h} \nabla \cdot \mathbf{z}_{h} d \Omega=0 .
$$

To prove the last GDP property (2.28), we will need the solution $(\phi, \mathbf{w})$ of the first-order problem

$$
\left\{\begin{array}{c}
\nabla \cdot \mathbf{w}=\nabla \cdot \mathbf{u}_{h} \quad \text { and } \quad \mathbf{w}+\nabla \phi=\mathbf{0} \quad \text { in } \Omega, \\
\phi=0 \quad \text { on } \Gamma_{D} \quad \text { and } \quad \mathbf{w} \cdot \mathbf{n}=0 \quad \text { on } \Gamma_{N} .
\end{array}\right.
$$

It will be also necessary to assume that this problem has full elliptic regularity, i.e., $\mathbf{w} \in \mathbf{H}^{1}(\Omega)$ and $\phi \in H^{2}(\Omega)$. Lastly, we recall the a priori bounds

$$
\|\mathbf{w}\|_{0} \leq\left\|\nabla \cdot \mathbf{u}_{h}\right\|_{-1} \quad \text { and } \quad\|\mathbf{w}\|_{1} \leq\left\|\nabla \cdot \mathbf{u}_{h}\right\|_{0} .
$$

Then, from $(2.22)$

$$
\left\|\mathbf{w}-\mathbf{w}_{h}\right\|_{0} \leq C h\|\mathbf{w}\|_{1} .
$$

Using this error estimate, the a priori bounds, and the triangle inequality yields that

$$
\begin{aligned}
\left\|\mathbf{w}_{h}\right\|_{0} & \leq\left\|\mathbf{w}_{h}-\mathbf{w}\right\|_{0}+\|\mathbf{w}\|_{0} \\
& \leq C h\|\mathbf{w}\|_{1}+\left\|\nabla \cdot \mathbf{u}_{h}\right\|_{-1} \leq C h\left\|\nabla \cdot \mathbf{u}_{h}\right\|_{0}+\left\|\nabla \cdot \mathbf{u}_{h}\right\|_{-1} .
\end{aligned}
$$

It was shown in [17] that the GDP, i.e., (2.25)-(2.28), along with the relation $\mathcal{W}_{k}^{3}(\Omega)=\nabla \cdot\left(\mathcal{W}_{k}^{2}(\Omega)\right)$, are necessary and sufficient for the stability of a mixed finite element method based on the Kelvin principle.

3. Least-squares finite element methods. A least-squares finite element method for the Poisson equation replaces the search for saddle-points of the Lagrangian functional, either $L_{D}(\phi, \mathbf{w}, \mathbf{u}, f)$ or $L_{K}(\lambda, \mathbf{u}, \phi, f)$, by a search for the unconstrained global minimizer of the quadratic functional

$$
J(\phi, \mathbf{u} ; f)=\frac{1}{2}\left(\|\nabla \cdot \mathbf{u}+\gamma \phi-f\|_{0}^{2}+\|\nabla \phi+\mathbf{u}\|_{0}^{2}\right) .
$$

The least-squares variational principle

$$
\min _{(\phi, \mathbf{u}) \in H_{D}^{1}(\Omega) \times H_{N}(\Omega, \operatorname{div})} J(\phi, \mathbf{u} ; f)
$$

then has a solution that minimizes the $L^{2}$ residuals of the first-order system (2.5). It is clear that this solution coincides with the solution of (2.5) or, equivalently, (1.1), and that it can be determined from the following first-order optimality system for (3.2): seek $(\phi, \mathbf{u}) \in H_{D}^{1}(\Omega) \times H_{N}(\Omega, \operatorname{div})$ such that

$$
Q((\phi, \mathbf{u}) ;(\psi, \mathbf{v}))=\mathcal{F}(\psi, \mathbf{v}) \quad \forall(\psi, \mathbf{v}) \in H_{D}^{1}(\Omega) \times H_{N}(\Omega, \operatorname{div}),
$$


where

$$
\begin{aligned}
Q((\phi, \mathbf{u}) ;(\psi, \mathbf{v}))= & \int_{\Omega}(\nabla \cdot \mathbf{u}+\gamma \phi)(\nabla \cdot \mathbf{v}+\gamma \psi) d \Omega \\
& +\int_{\Omega}(\nabla \phi+\mathbf{u}) \cdot(\nabla \psi+\mathbf{v}) d \Omega
\end{aligned}
$$

and

$$
\mathcal{F}(\psi, \mathbf{v})=\int_{\Omega} f(\nabla \cdot \mathbf{v}+\gamma \psi) d \Omega .
$$

To define a least-squares finite element method, we restrict (3.2) to the conforming subspace $\mathcal{W}_{m}^{0}(\Omega) \times \mathcal{W}_{k}^{2}(\Omega) \subset H_{D}^{1}(\Omega) \times H_{N}(\Omega$, div $)$. The least-squares finite element approximation is then obtained from the following discrete optimality system: seek $\left(\phi_{h}, \mathbf{u}_{h}\right) \in \mathcal{W}_{m}^{0}(\Omega) \times \mathcal{W}_{k}^{2}(\Omega)$ such that

$$
Q\left(\left(\phi_{h}, \mathbf{u}_{h}\right) ;\left(\psi_{h}, \mathbf{v}_{h}\right)\right)=\mathcal{F}\left(\psi_{h}, \mathbf{v}_{h}\right) \quad \forall\left(\psi_{h}, \mathbf{v}_{h}\right) \in \mathcal{W}_{m}^{0}(\Omega) \times \mathcal{W}_{k}^{2}(\Omega) .
$$

The next theorem states that

$$
\left\||\psi, \mathbf{v} \||=(Q((\psi, \mathbf{v}) ;(\psi, \mathbf{v})))^{1 / 2}\right.
$$

is an equivalent norm on $H_{D}^{1}(\Omega) \times H_{N}(\Omega$, div $)$. We call it the energy norm corresponding to the least-squares principle.

THEOREM 3.1. There exist positive constants $C_{1}$ and $C_{2}$ such that for any $(\psi, \mathbf{v}) \in H_{D}^{1}(\Omega) \times H_{N}(\Omega, \operatorname{div})$,

$$
C_{1}\left(\|\psi\|_{1}^{2}+\|\mathbf{v}\|_{H(\Omega, \text { div })}^{2}\right) \leq\|\| \psi, \mathbf{v}\|\|^{2} \leq C_{2}\left(\|\psi\|_{1}^{2}+\|\mathbf{v}\|_{H(\Omega, \text { div })}^{2}\right) .
$$

For a proof, see any of $[12,13,14,27]$. Theorem 3.1 implies that both the continuous variational problem (3.3) and its finite element restriction (3.6) are uniquely solvable and that their solutions are bounded by the norm of the data.

Note for later use that (3.3) and (3.6) imply the standard finite element orthogonality relation

$$
Q\left(\left(\phi-\phi_{h}, \mathbf{u}-\mathbf{u}_{h}\right) ;\left(\psi_{h}, \mathbf{v}_{h}\right)\right)=0 \quad \forall\left(\psi_{h}, \mathbf{v}_{h}\right) \in \mathcal{W}_{m}^{0}(\Omega) \times \mathcal{W}_{k}^{2}(\Omega) .
$$

3.1. Error estimates in $\boldsymbol{H}^{\mathbf{1}}(\Omega) \times \boldsymbol{H}(\Omega$, div $)$. In this section, we review the convergence properties of least-squares finite element methods for the Poisson equation with respect to the $H^{1}(\Omega) \times H(\Omega$, div $)$ norm. Most of the details are omitted, as the proofs follow by standard elliptic finite element arguments.

THEOREM 3.2. Assume that the solution $(\phi, \mathbf{u})$ of $(3.3)$ satisfies $(\phi, \mathbf{u}) \in H_{D}^{1}(\Omega) \cap$ $H^{m+1}(\Omega) \times H_{N}(\Omega, \operatorname{div}) \cap \mathbf{H}^{k+1}(\Omega)$ for some integers $k, m \geq 1$. Let $\left(\phi_{h}, \mathbf{u}_{h}\right) \in$ $\mathcal{W}_{m}^{0}(\Omega) \times \mathcal{W}_{k}^{2}(\Omega)$ be the solution of the least-squares finite element problem (3.6). Then, there exists a constant $C>0$ such that

$$
\left\|\phi-\phi_{h}\right\|_{1}+\left\|\mathbf{u}-\mathbf{u}_{h}\right\|_{H(\Omega, \operatorname{div})} \leq C\left(h^{k}\|\mathbf{u}\|_{k+1}+h^{m}\|\phi\|_{m+1}\right) .
$$

The error estimate (3.9) remains valid when $\mathbf{u}_{h}$ is approximated by the $C^{0}$ space $\left(P_{k}(\Omega)\right)^{n}$. 
Proof. Since $\left(\phi_{h}, \mathbf{u}_{h}\right)$ is a projection with respect to the energy norm $\||\cdot|\|$,

$$
\left\|\left|\phi-\phi_{h} ; \mathbf{u}-\mathbf{u}_{h}\|\mid \leq\| \phi-\psi_{h} ; \mathbf{u}-\mathbf{v}_{h}\|\| \quad \forall \psi_{h} \in \mathcal{W}_{m}^{0}(\Omega), \mathbf{v}_{h} \in \mathcal{W}_{k}^{2}(\Omega) .\right.\right.
$$

Then, (3.9) easily follows from the norm equivalence relation (3.7) and the approximation theoretic estimates (2.11)-(2.15).

Theorem 3.2 shows that the errors in $\mathbf{u}_{h}$ and $\phi_{h}$ will be equilibrated whenever $k=m$. For example, if any of the pairs $\left(R T_{0}, P_{1}\right),\left(B D M_{1}, P_{1}\right)$, or $\left(\left(P_{1}\right)^{n}, P_{1}\right)$ are used in the least-squares finite element method, the a priori bound (3.9) specializes to

$$
\left\|\phi-\phi_{h}\right\|_{1}+\left\|\mathbf{u}-\mathbf{u}_{h}\right\|_{H(\Omega, \text { div })} \leq C h\left(\|\mathbf{u}\|_{2}+\|\phi\|_{2}\right) .
$$

Therefore, the asymptotic accuracy of all three pairs in the norm of $H^{1}(\Omega) \times H(\Omega$, div) is the same. For this reason, in the implementation of the least-squares finite element method, one usually chooses the $C^{0}$ pair $\left(\left(P_{1}\right)^{n}, P_{1}\right)$ because it is the easiest to implement. Indeed, the ability to use equal-order interpolation has been often cited as a primary reason for choosing to use least-squares finite element methods. Nevertheless, the $C^{0}$ pair is not flawless because optimal $L^{2}$ norm errors for the flux approximation have proven impossible to obtain without augmenting (2.5) with an additional redundant curl constraint equation. Also, as we have already mentioned, numerical studies in [16] indicate that the $L^{2}$ convergence of the flux is indeed suboptimal with $C^{0}$ finite element spaces.

The curl constraint, first introduced in the least-squares finite element setting in [15] and subsequently utilized by many others (see, e.g., [12, 13, 14, 25]), makes the least-squares functional norm-equivalent on $H^{1}(\Omega) \times \mathbf{H}^{1}(\Omega)$. However, in some situations the curl equation may unduly restrict the range of the differential operator and should be avoided. In the next section, we will see that if the nodal approximation of the flux is replaced by an approximation in $\mathcal{W}_{k}^{2}(\Omega)$, it may be possible to recover optimal $L^{2}$ convergence rates without adding the curl constraint. As in [16], the key to this is the GDP.

3.2. Error estimates in $\boldsymbol{L}^{2}$. Throughout this section, we let $(\phi, \mathbf{u})$ and $\left(\phi_{h}, \mathbf{u}_{h}\right)$ $\in \mathcal{W}_{m}^{0}(\Omega) \times \mathcal{W}_{k}^{2}(\Omega)$ denote the solutions of (3.3) and (3.6), respectively. We assume that the solution of the problem

$$
-\Delta \psi+\gamma \psi=\eta \quad \text { in } \Omega, \quad \psi=0 \quad \text { on } \Gamma_{D}, \quad \frac{\partial \psi}{\partial n}=0 \quad \text { on } \Gamma_{D}
$$

satisfies the regularity estimate

$$
\|\psi\|_{s+2} \leq C\|\eta\|_{s} \text { for } s=0,1 \text { and } \forall \eta \in H^{s}(\Omega) .
$$

This additional regularity is necessary since our $L^{2}$ error estimates are based on a duality argument.

3.2.1. $L^{2}$ error estimates for the scalar variable. Our first lemma bounds the negative norm of the error in the first equation in (2.5) in terms of the energy norm of the total error. Note that (3.11) of course implies that $\|\nabla \psi\|_{s+1} \leq C\|\eta\|_{s}$ for $s=0,1$.

LEMmA 3.3. Let $\left(\phi_{h}, \mathbf{u}_{h}\right)$ be a least-squares finite element approximation of $(\phi, \mathbf{u})$. Then,

$$
\left\|\nabla \cdot\left(\mathbf{u}-\mathbf{u}_{h}\right)+\gamma\left(\phi-\phi_{h}\right)\right\|_{-1} \leq C h\|\| \phi-\phi_{h}, \mathbf{u}-\mathbf{u}_{h}\|\| .
$$


Proof. Let $\eta \in H_{0}^{1}(\Omega)$ be an arbitrary function, let $\psi$ solve the boundary value problem (3.10), and let $\mathbf{v}=-\nabla \psi$. One then obtains

$$
\begin{aligned}
\int_{\Omega}\left(\nabla \cdot\left(\mathbf{u}-\mathbf{u}_{h}\right)+\gamma\left(\phi-\phi_{h}\right)\right) \eta d \Omega \\
=\int_{\Omega}\left(\nabla \cdot\left(\mathbf{u}-\mathbf{u}_{h}\right)+\gamma\left(\phi-\phi_{h}\right)\right)(\nabla \cdot \mathbf{v}+\gamma \psi) d \Omega \\
=Q\left(\phi-\phi_{h}, \mathbf{u}-\mathbf{u}_{h} ; \psi, \mathbf{v}\right)=Q\left(\phi-\phi_{h}, \mathbf{u}-\mathbf{u}_{h} ; \psi-\mathcal{I}_{0} \psi, \mathbf{v}-\mathcal{I}_{2} \mathbf{v}\right) \\
=\quad \int_{\Omega}\left(\nabla \cdot\left(\mathbf{u}-\mathbf{u}_{h}\right)+\gamma\left(\phi-\phi_{h}\right)\right)\left(\nabla \cdot\left(\mathbf{v}-\mathcal{I}_{2} \mathbf{v}\right)+\gamma\left(\psi-\mathcal{I}_{0} \psi\right) d \Omega\right. \\
\quad \quad \quad \int_{\Omega}\left(\nabla\left(\phi-\phi_{h}\right)+\left(\mathbf{u}-\mathbf{u}_{h}\right)\right) \cdot\left(\nabla\left(\psi-\mathcal{I}_{0} \psi\right)+\left(\mathbf{v}-\mathcal{I}_{2} \mathbf{v}\right)\right) d \Omega \\
\leq C\left(\left(\left\|\nabla \cdot\left(\mathbf{u}-\mathbf{u}_{h}\right)\right\|_{0}+\left\|\phi-\phi_{h}\right\|_{0}\right)\left(\left\|\nabla \cdot\left(\mathbf{v}-\mathcal{I}_{2} \mathbf{v}\right)\right\|_{0}+\left\|\psi-\mathcal{I}_{0} \psi\right\|_{0}\right)\right. \\
\left.\quad \quad+\left(\left\|\nabla\left(\phi-\phi_{h}\right)\right\|_{0}+\left\|\mathbf{u}-\mathbf{u}_{h}\right\|_{0}\right)\left(\left\|\nabla\left(\psi-\mathcal{I}_{0} \psi\right)\right\|_{0}+\left\|\mathbf{v}-\mathcal{I}_{2} \mathbf{v}\right\|_{0}\right)\right),
\end{aligned}
$$

where we have successively used $\nabla \psi+\mathbf{v}=0$, the error orthogonality (3.8), the definition of $Q(\cdot, \cdot ; \cdot, \cdot)$, and the Cauchy-Schwarz inequality. Using the interpolation error estimates (2.11)-(2.15) and the regularity assumption (3.11), we have that

$$
\left.\begin{array}{r}
\left\|\nabla \cdot\left(\mathbf{v}-\mathcal{I}_{2} \mathbf{v}\right)\right\|_{0} \leq C h\|\mathbf{v}\|_{2} \leq C h\|\eta\|_{1} \\
\left\|\mathbf{v}-\mathcal{I}_{2} \mathbf{v}\right\|_{0} \leq C h\|\mathbf{v}\|_{2} \leq C h\|\eta\|_{1} \\
\left\|\nabla\left(\psi-\mathcal{I}_{0} \psi\right)\right\|_{0} \leq C h\|\psi\|_{2} \leq C h\|\eta\|_{1} \\
\left\|\psi-\mathcal{I}_{0} \psi\right\|_{0} \leq C h^{2}\|\psi\|_{2} \leq C h^{2}\|\eta\|_{1}
\end{array}\right\} \quad \forall \eta \in H_{0}^{1}(\Omega) .
$$

Combining the last two sets of results, we easily obtain that, for all $\eta \in H_{0}^{1}(\Omega)$,

$$
\int_{\Omega}\left(\nabla \cdot\left(\mathbf{u}-\mathbf{u}_{h}\right)+\gamma\left(\phi-\phi_{h}\right)\right) \eta d \Omega \leq C h\left(\left\|\phi-\phi_{h}\right\|_{1}+\left\|\mathbf{u}-\mathbf{u}_{h}\right\|_{H(\Omega, \operatorname{div})}\right)\|\eta\|_{1},
$$

while the left inequality in (3.7) gives

$$
\int_{\Omega}\left(\nabla \cdot\left(\mathbf{u}-\mathbf{u}_{h}\right)+\gamma\left(\phi-\phi_{h}\right)\right) \eta d \Omega \leq C h\left|\left\|\phi-\phi_{h}, \mathbf{u}-\mathbf{u}_{h}\left|\|\mid\| \eta \|_{1} \quad \forall \eta \in H_{0}^{1}(\Omega) .\right.\right.\right.
$$

The lemma follows by taking a supremum over $\eta \in H_{0}^{1}(\Omega)$.

Next, we bound the $L^{2}$ error in $\phi_{h}$ by the energy norm. Then,

Lemma 3.4. Let $\left(\phi_{h}, \mathbf{u}_{h}\right)$ be a least-squares finite element approximation of $(\phi, \mathbf{u})$.

$$
\left\|\phi-\phi_{h}\right\|_{0} \leq C h\left|\left\|\phi-\phi_{h}, \mathbf{u}-\mathbf{u}_{h} \mid\right\| .\right.
$$

Proof. Let $\psi$ solve the boundary value problem

$$
\begin{cases}-\triangle \psi+\gamma \psi=\phi-\phi_{h} & \text { in } \Omega, \\ \psi=0 \text { on } \Gamma_{D} \quad \text { and } & \partial \psi / \partial n=0 \text { on } \Gamma_{N} .\end{cases}
$$

The regularity assumption (3.11) implies that

$$
\|\psi\|_{2} \leq C\left\|\phi-\phi_{h}\right\|_{0} .
$$


Using the definition of $\psi$, integration by parts, and the definition of the least-squares form (3.4) yields

$$
\begin{aligned}
\left\|\phi-\phi_{h}\right\|_{0}^{2}= & \int_{\Omega}\left(\phi-\phi_{h}\right)(-\triangle \psi+\gamma \psi) d \Omega=\int_{\Omega}\left(\nabla\left(\phi-\phi_{h}\right) \cdot \nabla \psi+\gamma\left(\phi-\phi_{h}\right) \psi\right) d \Omega \\
= & \int_{\Omega}\left(\nabla\left(\phi-\phi_{h}\right)+\left(\mathbf{u}-\mathbf{u}_{h}\right)\right) \cdot \nabla \psi d \Omega \\
& -\int_{\Omega}\left(\mathbf{u}-\mathbf{u}_{h}\right) \cdot \nabla \psi d \Omega+\int_{\Omega} \gamma\left(\phi-\phi_{h}\right) \psi d \Omega \\
= & Q\left(\phi-\phi_{h}, \mathbf{u}-\mathbf{u}_{h} ; \psi, 0\right)+\int_{\Omega}(1-\gamma)\left(\nabla \cdot\left(\mathbf{u}-\mathbf{u}_{h}\right)+\gamma\left(\phi-\phi_{h}\right)\right) \psi d \Omega .
\end{aligned}
$$

Using the error orthogonality (3.8), the definition (3.14) for $\psi$, and the regularity assumption (3.15) yields

$$
\begin{aligned}
& Q\left(\phi-\phi_{h}, \mathbf{u}-\mathbf{u}_{h} ; \psi, 0\right)=Q\left(\phi-\phi_{h}, \mathbf{u}-\mathbf{u}_{h} ; \psi-\mathcal{I}_{0} \psi, 0\right) \\
& \quad \leq\|\| \phi-\phi_{h}, \mathbf{u}-\mathbf{u}_{h}\left|\left\|\left|\left\|\psi-\mathcal{I}_{0} \psi\right\|_{1} \leq C h\left\|\mid \phi-\phi_{h}, \mathbf{u}-\mathbf{u}_{h}\right\|\|\| \psi \|_{2}\right.\right.\right. \\
& \quad \leq C h\left\||| \phi-\phi_{h}, \mathbf{u}-\mathbf{u}_{h}\right\| \mid\left\|\phi-\phi_{h}\right\|_{0} .
\end{aligned}
$$

In addition, the definition (3.14) for $\psi,(3.15)$, and (3.12) imply that

$$
\begin{aligned}
& \int_{\Omega}(1-\gamma)\left(\nabla \cdot\left(\mathbf{u}-\mathbf{u}_{h}\right)+\gamma\left(\phi-\phi_{h}\right)\right) \psi d \Omega \leq\left\|\nabla \cdot\left(\mathbf{u}-\mathbf{u}_{h}\right)+\gamma\left(\phi-\phi_{h}\right)\right\|_{-1}\|\psi\|_{1} \\
& \quad \leq C\left\|\nabla \cdot\left(\mathbf{u}-\mathbf{u}_{h}\right)+\gamma\left(\phi-\phi_{h}\right)\right\|_{-1}\left\|\phi-\phi_{h}\right\|_{0} \\
& \quad \leq C h\left\|\phi-\phi_{h}, \mathbf{u}-\mathbf{u}_{h}\right\| \mid\left\|\phi-\phi_{h}\right\|_{0} .
\end{aligned}
$$

The lemma easily follows by combining the last three results.

Corollary 3.5. Assume that the regularity assumption (3.11) is satisfied, and assume that the solution $(\phi, \mathbf{u})$ of $(3.3)$ satisfies $(\phi, \mathbf{u}) \in H_{D}^{1}(\Omega) \cap H^{m+1}(\Omega) \times$ $H_{N}(\Omega$, div $) \cap \mathbf{H}^{k+1}(\Omega)$ for some integers $k, m \geq 1$. Let $\left(\phi_{h}, \mathbf{u}_{h}\right) \in \mathcal{W}_{m}^{0}(\Omega) \times \mathcal{W}_{k}^{2}(\Omega)$ be the solution of the least-squares finite element problem (3.6). Then, there exists a constant $C>0$ such that

$$
\left\|\phi-\phi_{h}\right\|_{0} \leq C\left(h^{k+1}\|\mathbf{u}\|_{k+1}+h^{m+1}\|\phi\|_{m+1}\right) .
$$

Proof. The corollary follows simply by a direct application of (3.7) and (3.9) to (3.13).

The optimal $L^{2}$ error bound (3.16) for the scalar variable does not depend on whether or not the finite element space for the flux satisfies (2.25)-(2.28), i.e., the GDP. Thus, it remains valid even when equal-order $C^{0}$ finite element functions are used for the flux approximations, a result first shown in [24]. On the other hand, we will see that the GDP is needed if one wants to improve the $L^{2}$ accuracy of the flux.

3.2.2. $L^{2}$ error estimate for the flux. Ultimately, the final $L^{2}$ error estimates for approximations to the flux depend on whether $\mathcal{W}_{k}^{2}(\Omega)$ represents the $\mathrm{RT}_{k-1}$ or the $\mathrm{BDM}_{k}$ family. To this end, we need the following result.

Lemma 3.6. Let $\left(\phi_{h}, \mathbf{u}_{h}\right) \in \mathcal{W}_{m}^{0}(\Omega) \times \mathcal{W}_{k}^{2}(\Omega)$ be the least-squares finite element approximation defined by $(3.6)$. Then,${ }^{14}$

$$
\left\|\mathbf{u}-\mathbf{u}_{h}\right\|_{0} \leq C\left(h \left\|\left|\phi-\phi_{h}, \mathbf{u}-\mathbf{u}_{h}\|\mid+h\| \nabla \cdot\left(\mathbf{u}-\mathbf{v}_{h}\right)\left\|_{0}+\right\| \mathbf{u}-\mathbf{v}_{h} \|_{0}\right)\right.\right.
$$

\footnotetext{
${ }^{14}$ It is clear from the proof of this lemma that it holds for any flux approximation that satisfies the GDP.
} 
for any $\mathbf{v}_{h} \in \mathcal{W}_{k}^{2}(\Omega)$.

Proof. Let $\mathbf{v}_{h}$ be an arbitrary element of $\mathcal{W}_{k}^{2}(\Omega)$. From Theorem 2.1, we know that there exist $\mathbf{z}_{h}$ and $\mathbf{w}_{h}$, also in $\mathcal{W}_{k}^{2}(\Omega)$, such that

$$
\mathbf{u}_{h}-\mathbf{v}_{h}=\mathbf{w}_{h}+\mathbf{z}_{h}
$$

and the properties (2.26)-(2.28) hold. We recall for later use that

$$
\|\nabla \cdot \mathbf{v}\|_{-1} \leq\|\mathbf{v}\|_{0} \quad \forall \mathbf{v} \in H_{N}(\Omega, \operatorname{div}) .
$$

We now bound the two GDP components of $\mathbf{u}_{h}-\mathbf{v}_{h}$ in $L^{2}$. To estimate $\left\|\mathbf{w}_{h}\right\|_{0}$, we successively use (2.28), (3.18), (3.12), (3.7), and (3.13) to obtain

$$
\begin{aligned}
&\left\|\mathbf{w}_{h}\right\|_{0} \leq C\left(\left\|\nabla \cdot\left(\mathbf{u}_{h}-\mathbf{v}_{h}\right)\right\|_{-1}+h\left\|\nabla \cdot\left(\mathbf{u}_{h}-\mathbf{v}_{h}\right)\right\|_{0}\right) \\
& \leq C\left(\left\|\nabla \cdot\left(\mathbf{u}-\mathbf{u}_{h}\right)\right\|_{-1}+h\left\|\nabla \cdot\left(\mathbf{u}-\mathbf{u}_{h}\right)\right\|_{0}\right. \\
&\left.\quad+\left\|\nabla \cdot\left(\mathbf{u}-\mathbf{v}_{h}\right)\right\|_{-1}+h\left\|\nabla \cdot\left(\mathbf{u}-\mathbf{v}_{h}\right)\right\|_{0}\right) \\
& \leq \quad C\left(\left\|\nabla \cdot\left(\mathbf{u}-\mathbf{u}_{h}\right)+\gamma\left(\phi-\phi_{h}\right)\right\|_{-1}+h\left\|\nabla \cdot\left(\mathbf{u}-\mathbf{u}_{h}\right)\right\|_{0}\right. \\
&\left.\quad+\left\|\gamma\left(\phi-\phi_{h}\right)\right\|_{-1}+\left\|\mathbf{u}-\mathbf{v}_{h}\right\|_{0}+h\left\|\nabla \cdot\left(\mathbf{u}-\mathbf{v}_{h}\right)\right\|_{0}\right) \\
& \leq C\left(h\left\|\phi-\phi_{h}, \mathbf{u}-\mathbf{u}_{h}\right\|\|+\| \phi-\phi_{h}\left\|_{0}+\right\| \mathbf{u}-\mathbf{v}_{h}\left\|_{0}+h\right\| \nabla \cdot\left(\mathbf{u}-\mathbf{v}_{h}\right) \|_{0}\right) \\
& \leq C\left(h\left\|\phi-\phi_{h}, \mathbf{u}-\mathbf{u}_{h}\right\| \mid+\left\|\mathbf{u}-\mathbf{v}_{h}\right\|_{0}+h\left\|\nabla \cdot\left(\mathbf{u}-\mathbf{v}_{h}\right)\right\|_{0}\right) .
\end{aligned}
$$

To estimate $\left\|\mathbf{z}_{h}\right\|_{0}$, we use the error orthogonality (3.8) with $\psi_{h}=0$ and $\mathbf{v}_{h}=\mathbf{z}_{h}$. Since from (2.26) we have that $\nabla \cdot \mathbf{z}_{h}=0$, this identity reduces to

$$
\int_{\Omega}\left(\nabla\left(\phi-\phi_{h}\right)+\left(\mathbf{u}-\mathbf{u}_{h}\right)\right) \cdot \mathbf{z}_{h} d \Omega=0
$$

from which integrating by parts and again using $\nabla \cdot \mathbf{z}_{h}=0$ yields

$$
\int_{\Omega}\left(\mathbf{u}-\mathbf{u}_{h}\right) \cdot \mathbf{z}_{h} d \Omega=0 .
$$

Using this result and the orthogonality of $\mathbf{z}_{h}$ and $\mathbf{w}_{h}$ (see (2.27)), we obtain

$$
\begin{aligned}
\left\|\mathbf{z}_{h}\right\|_{0}^{2} & =\int_{\Omega} \mathbf{z}_{h} \cdot \mathbf{z}_{h} d \Omega=\int_{\Omega}\left(\mathbf{z}_{h}+\mathbf{w}_{h}\right) \cdot \mathbf{z}_{h} d \Omega \\
& =\int_{\Omega}\left(\mathbf{u}_{h}-\mathbf{v}_{h}\right) \cdot \mathbf{z}_{h} d \Omega=\int_{\Omega}\left(\mathbf{u}-\mathbf{v}_{h}\right) \cdot \mathbf{z}_{h} d \Omega
\end{aligned}
$$

so that

$$
\left\|\mathbf{z}_{h}\right\|_{0} \leq\left\|\mathbf{u}-\mathbf{v}_{h}\right\|_{0} .
$$

To complete the proof, we note that

$$
\left\|\mathbf{u}-\mathbf{u}_{h}\right\|_{0} \leq\left\|\mathbf{u}-\mathbf{v}_{h}\right\|_{0}+\left\|\mathbf{u}_{h}-\mathbf{v}_{h}\right\|_{0} \leq\left\|\mathbf{u}-\mathbf{v}_{h}\right\|_{0}+\left\|\mathbf{w}_{h}\right\|_{0}+\left\|\mathbf{z}_{h}\right\|_{0}
$$

and then use the bounds on $\mathbf{z}_{h}$ and $\mathbf{w}_{h}$. 
Let us now inspect (3.17). The first term on the right-hand side is exactly the same one as in the $L^{2}$ bound (3.13) for the scalar variable. Let us further assume that the approximation orders of the spaces used for the scalar variable and the flux are equilibrated, i.e., $\phi_{h} \in \mathcal{W}_{r}^{0}(\Omega)$ and $\mathbf{u}_{h} \in \mathcal{W}_{r}^{2}(\Omega)$ for some $r \geq 1$. Then,

$$
\left\|\left|\phi-\phi_{h}, \mathbf{u}-\mathbf{u}_{h} \|\right| \leq C h^{r}\left(\|\phi\|_{r+1}+\|\mathbf{u}\|_{r+1}\right) .\right.
$$

The additional factor $h$ multiplying this term in (3.17) will increase the order of that term to $r+1$, just as in (3.16). However, (3.17) contains the two additional terms $h\left\|\nabla \cdot\left(\mathbf{u}-\mathbf{v}_{h}\right)\right\|_{0}$ and $\left\|\mathbf{u}-\mathbf{v}_{h}\right\|_{0}$. Recall that $\mathcal{W}_{r}^{2}(\Omega)$ represents RT and BDM spaces that are equilibrated with respect to the divergence error. Therefore, after setting $\mathbf{v}_{h}=\mathcal{I}_{2} \mathbf{u}$, from (2.15) it follows that

$$
\left\|\nabla \cdot\left(\mathbf{u}-\mathbf{v}_{h}\right)\right\|_{0} \leq C h^{r}\|\mathbf{u}\|_{r+1} .
$$

After multiplication by $h$, the order of this term also increases to $r+1$. However, the order of the last term will depend on whether $\mathcal{W}_{r}^{2}(\Omega)$ represents a BDM or RT space. Indeed, from (2.16),

$$
\left\|\mathbf{u}-\mathbf{v}_{h}\right\|_{0} \leq C \begin{cases}h^{r}\|\mathbf{u}\|_{r} & \text { if } \mathcal{W}_{r}^{2}(\Omega)=\mathrm{RT}_{r-1}, \\ h^{r+1}\|\mathbf{u}\|_{r+1} & \text { if } \mathcal{W}_{r}^{2}(\Omega)=\mathrm{BDM}_{r}\end{cases}
$$

The next corollary summarizes these observations.

COROLlary 3.7. Assume that the regularity assumption (3.11) is satisfied, and assume that the solution $(\phi, \mathbf{u})$ of $(3.3)$ satisfies $(\phi, \mathbf{u}) \in H_{D}^{1}(\Omega) \cap H^{r+1}(\Omega) \times$ $H_{N}(\Omega$, div $) \cap \mathbf{H}^{r+1}(\Omega)$ for some integer $r \geq 1$. Let $\left(\phi_{h}, \mathbf{u}_{h}\right) \in \mathcal{W}_{r}^{0}(\Omega) \times \mathcal{W}_{r}^{2}(\Omega)$ be the solution of the least-squares finite element problem (3.6). Then, there exists a constant $C>0$ such that

$$
\left\|\mathbf{u}-\mathbf{u}_{h}\right\|_{0} \leq C \begin{cases}h^{r}\left(\|\mathbf{u}\|_{r+1}+\|\phi\|_{r+1}\right) & \text { if } \mathcal{W}_{r}^{2}(\Omega)=\mathrm{RT}_{r-1} \\ h^{r+1}\left(\|\mathbf{u}\|_{r+1}+\|\phi\|_{r+1}\right) & \text { if } \mathcal{W}_{r}^{2}(\Omega)=\mathrm{BDM}_{r}\end{cases}
$$

Consider, for example, the lowest-order case for which $r=1, \mathcal{W}_{1}^{0}(\Omega)=P_{1}$, and $\mathcal{W}_{1}^{2}(\Omega)$ is either $\mathrm{RT}_{0}$ or $\mathrm{BDM}_{1}$. If the least-squares finite element method is implemented with $\mathrm{RT}_{0}$ elements, (3.19) specializes to

$$
\left\|\mathbf{u}-\mathbf{u}_{h}\right\|_{0} \leq h\left(\|\mathbf{u}\|_{2}+\|\phi\|_{2}\right) .
$$

If instead we use $\mathrm{BDM}_{1}$ elements, we then obtain the improved error bound

$$
\left\|\mathbf{u}-\mathbf{u}_{h}\right\|_{0} \leq h^{2}\left(\|\mathbf{u}\|_{2}+\|\phi\|_{2}\right)
$$

It is worth repeating that the reason for this difference in the $L^{2}$ errors is the structure of the RT spaces. Since $\mathrm{RT}_{0}=\left(P_{0}\right)^{n}+\mathbf{x} P_{0}$, the approximation properties of $\mathrm{RT}_{0}$ in $L^{2}$ are the same as those of $P_{0}$. However, it is easy to see that, thanks to the extra term $\mathbf{x} P_{0}, \nabla \cdot\left(\mathrm{RT}_{0}\right)=P_{0}$; i.e., the divergence of $\mathbf{u}$ is approximated to the same order as the field itself. For numerical examples with least-squares methods that illustrate this feature of RT spaces, we refer to [2]. 
4. Least-squares finite element methods and duality. We already saw that a least-squares finite element method implemented using equal-order $C^{0}$ finite element spaces approximates the scalar variable with the same accuracy as a Galerkin (or, equivalently, a mixed) method for the Dirichlet principle. However, the approximation properties of the Kelvin principle are only partially inherited in the sense that the accuracy in the approximation to the divergence of the flux is recovered, but the accuracy in the flux approximation itself may be of one order less. This should not be too much of a surprise because $C^{0}$ elements provide stable discretization only for the Dirichlet principle (with the exception of the criss-cross grid; see [16]). While least-squares minimization is stable enough to allow for the approximation of scalar variables and the flux by equal-order $C^{0}$ finite element spaces, it cannot completely recover from the fact that such spaces are unstable for the Kelvin principle.

The key observation from section 3.2 is that a least-squares finite element method can inherit the computational properties of both the Dirichlet and the Kelvin principles, provided the scalar variable and the flux are approximated by finite element spaces that are stable with respect to these two principles. Then, as our analysis showed, least-squares finite element solutions recover the accuracy of the Dirichlet principle for the scalar variable and the accuracy of the Kelvin principle for the flux.

In a way, we see that, implemented in this particular manner, the least-squares finite element method represents a balanced mixture of the two principles. Below, we provide an explanation of this observation using the apparatus of differential form calculus, albeit in a simplified form and without an explicit reference to differential forms on manifolds. For consistency, in what follows, $H(\Omega, \operatorname{grad}), H(\Omega$, curl $)$, and $H(\Omega$, div) denote spaces of square integrable functions whose gradients, curls, and divergences, respectively, are also square integrable. ${ }^{15}$

The De Rham differential complex

$$
\mathbb{R} \hookrightarrow H(\Omega, \operatorname{grad}) \stackrel{\nabla}{\longmapsto} H(\Omega, \text { curl }) \stackrel{\nabla \times}{\longmapsto} H(\Omega, \text { div }) \stackrel{\nabla \cdot}{\longmapsto} L^{2}(\Omega) \longmapsto 0
$$

is an exact sequence of spaces in the sense that each operator maps the space on its left to the kernel of the next operator in the sequence, and the last mapping is a surjection. We will now start to use the identifications

$$
\mathcal{W}^{0}(\Omega)=H(\Omega, \operatorname{grad}), \mathcal{W}^{1}(\Omega)=H(\Omega, \operatorname{curl}), \mathcal{W}^{2}(\Omega)=H(\Omega, \operatorname{div}), \mathcal{W}^{3}(\Omega)=L^{2}(\Omega)
$$

to indicate that these function spaces are comprised of proxies for differential forms of orders $0,1,2$, and 3, respectively. ${ }^{16}$ Exact sequences of finite element spaces provide piecewise polynomial approximations of the proxies. Commonly used terminologies for the finite element subspaces of $\mathcal{W}^{0}, \mathcal{W}^{1}, \mathcal{W}^{2}$, and $\mathcal{W}^{3}$ are nodal, edge, face, and volume (or discontinuous) elements, respectively.

Differential forms have always played a fundamental role in classical mechanics and numerical methods for Hamiltonian systems; see, e.g., $[3,6]$. Their place as an abstraction tool for discretization of elliptic boundary value problems was perhaps first recognized in [21], while [8, 9] further affirmed their importance in computational electromagnetism.

Subsequently, the idea that a stable partial differential equation discretization can be developed using a discrete equivalent of the De Rham complex has been exploited by many researchers in finite element, finite volume, and finite difference methods

\footnotetext{
${ }^{15}$ Here we treat the case of $n=3$; similar developments can be carried out for the two-dimensional case.

${ }^{16}$ This should explain the seemingly peculiar choice of notation introduced earlier in the paper.
} 
$[1,5,19,20,22,23,26]$; see [4] for a more extensive bibliography. In particular, for second-order elliptic problems, a key tool for encoding their structure is provided by the factorization or Tonti diagrams; see [19, 20]. Essentially, these diagrams break the problem into topological relations between different spaces in a De Rham complex connected by metric relations expressed by the Hodge *-operator, a linear map $\mathcal{W}^{k}(\Omega) \mapsto \mathcal{W}^{n-k}(\Omega)$. The factorization diagram for the Poisson problem (see, e.g., $[20,26])$ is

$$
\begin{array}{ccccccc}
\mathcal{W}^{0}(\Omega) & \phi & \stackrel{\nabla}{\longrightarrow} & -\mathbf{u} & & \mathcal{W}^{1}(\Omega) \\
& \xi=* \phi & \uparrow & & \uparrow & \mathbf{q}=* \mathbf{u} & \\
\mathcal{W}^{3}(\Omega) & & \mathrm{f}-\gamma \xi & \stackrel{\nabla}{\longleftarrow} & \mathbf{q} & & \mathcal{W}^{2}(\Omega)
\end{array}
$$

We will refer to the relation and the variables on the top of the diagram as the primal variables and equilibrium equation. The dual variables and their "equilibrium" equation are represented by the bottom part of the diagram. The dual and primal variables serve as proxies for 0,1 and 2, 3 differential forms, respectively.

The horizontal links in (4.2) correspond to the differential equations

$$
\nabla \phi=-\mathbf{u} \text { and } \nabla \cdot \mathbf{q}=-\gamma \xi+f
$$

while the vertical links provide the "constitutive" relations

$$
\xi=* \phi \quad \text { and } \quad \mathbf{q}=* \mathbf{u} .
$$

The importance of structures such as (4.2) stems from the fact that they encode fundamental relationships between spaces and operators that are required for the stability of discretizations; see, e.g., [1, 4, 9, 20, 22].

Let us now show that the Dirichlet and Kelvin principles are obtained from (4.2) by the approximation of the Hodge operator by an identity operator and subsequent elimination of the dual or the primal variables, respectively.

If the dual variables are substituted by the primal ones according to

$$
\xi=\phi \quad \text { and } \quad \mathbf{q}=\mathbf{u},
$$

then the dual equation in (4.2) must be modified to account for the fact that $\mathbf{u}$ is a proxy of a 1-form, rather than of a 2 -form. As such, $\mathbf{u}$ is in the domain of the curl operator but not in the domain of the divergence operator. Thus, in the dual equilibrium equation, we replace the divergence operator by a weak divergence operator defined through the following variational statement:

$$
\widetilde{\nabla} \cdot: \mathcal{W}^{1}(\Omega) \mapsto \mathcal{W}^{0}(\Omega), \quad \widetilde{\nabla} \cdot \mathbf{u}=\phi
$$

if and only if

$$
\int_{\Omega} \phi \psi d \Omega=-\int_{\Omega} \mathbf{u} \cdot \nabla \psi d \Omega \quad \forall \psi \in \mathcal{W}^{0}(\Omega)
$$

This changes the original factorization diagram to one in terms of only the primal variables:

$$
\begin{array}{ccccccc}
\mathcal{W}^{0}(\Omega) & & \phi & \stackrel{\nabla}{\longrightarrow} & -\mathbf{u} & & \mathcal{W}^{1}(\Omega) \\
& \eta=\phi & \downarrow & & \downarrow & \mathbf{v}=\mathbf{u} & \\
\mathcal{W}^{0}(\Omega) & & \mathrm{f}-\gamma \phi & \stackrel{\widetilde{\nabla}}{\longleftarrow} & \mathbf{u} & & \mathcal{W}^{1}(\Omega)
\end{array}
$$


The partial differential equation system represented by this diagram is ${ }^{17}$

$$
\begin{array}{rr}
\nabla \phi+\mathbf{u}=0 & \text { in } \mathcal{W}^{1}(\Omega), \\
-\int_{\Omega} \mathbf{u} \cdot \nabla \psi-\gamma \phi \psi d \Omega=\int_{\Omega} f \psi d \Omega & \forall \psi \in \mathcal{W}^{0}(\Omega) .
\end{array}
$$

One recognizes (4.3) as the optimality system (2.4) for the Dirichlet principle. The diagram (4.3) can be viewed as a representation of this principle.

If instead the primal variables are eliminated according to

$$
\phi=\xi \text { and } \quad \mathbf{u}=\mathbf{q},
$$

then the primal equilibrium equation in (4.2) must be modified to account for the fact that $\xi$ is a proxy of a 3 -form, rather than for a 0 -form. As such, $\xi$ is not in the domain of the gradient operator, which therefore must be replaced by a weak one:

$$
\widetilde{\nabla}: \mathcal{W}^{3}(\Omega), \mapsto \mathcal{W}^{2}(\Omega), \quad \widetilde{\nabla} \xi=\mathbf{q},
$$

if and only if

$$
\int_{\Omega} \mathbf{q} \cdot \mathbf{v} d \Omega=-\int_{\Omega} \xi \nabla \cdot \mathbf{v} d \Omega \quad \forall \mathbf{v} \in \mathcal{W}^{2}(\Omega)
$$

The factorization diagram in terms of the dual variables is then given by

$$
\begin{array}{ccccccc}
\mathcal{W}^{3}(\Omega) & & \xi & \stackrel{\widetilde{\nabla}}{\rightleftarrows} & -\mathbf{q} & & \mathcal{W}^{2}(\Omega) \\
& \phi=\xi & \uparrow & & \uparrow & \mathbf{u}=\mathbf{q} & \\
\mathcal{W}^{3}(\Omega) & & \mathrm{f}-\gamma \xi & \stackrel{\nabla}{\longleftarrow} & \mathbf{q} & & \mathcal{W}^{2}(\Omega)
\end{array}
$$

The problem represented by this diagram is

$$
\begin{array}{rr}
\int_{\Omega} \mathbf{q} \cdot \mathbf{v} d \Omega-\int_{\Omega} \xi \nabla \cdot \mathbf{v} d \Omega=0 & \forall \mathbf{v} \in \mathcal{W}^{2}(\Omega), \\
\nabla \cdot \mathbf{v}+\gamma \xi=f & \text { in } \mathcal{W}^{3}(\Omega) .
\end{array}
$$

Now the second equation is an exact relation, and we see that, by elimination of the primal variables, we recover the optimality system (2.10) for the Kelvin principle.

It is now clear that each of the classical variational principles for the system (1.1) can be derived from (4.2) by elimination of one of the sets of variables (primal or dual) and relaxation of the complementary equilibrium equation. Elimination of variables, on the other hand, can be interpreted as approximation of the Hodge $*$-operator by an identity. This, of course, immediately leads to the following question: what kinds of variational principles can be obtained by using other ways of approximating the Hodge operator? Here, we will focus on one particular method wherein this operator is replaced by an $L^{2}$ projection. Not surprisingly, we will see that this approximation leads eventually to a least-squares principle for the first-order formulation of (1.1), but one that is necessarily implemented with spaces for the scalar inherited from the

\footnotetext{
${ }^{17}$ The first equation can also be stated in variational form; see (2.4). However, we write it in algebraic form to stress the fact that it represents an exact relationship.
} 
Dirichlet principle, and, for the flux, from the Kelvin principle. Thus, in a sense, the least-squares method, when implemented in this manner, is indeed a mixture of the two classical principles that combines their best properties.

The idea is to keep both the primal and dual sets of variables, but to replace the Hodge operator by an optimization problem that penalizes the discrepancy between these sets. Then, the primal and dual equations become linear constraints that must be satisfied by the minimizers of this functional. Therefore, we are led to the following constrained optimization problem: seek $(\phi, \mathbf{u}, \xi, \mathbf{q})$ in $W^{0}(\Omega) \times \mathcal{W}^{1}(\Omega) \times \mathcal{W}^{2}(\Omega) \times$ $\mathcal{W}^{3}(\Omega)$ such that

$$
\mathcal{J}(\phi, \mathbf{u}, \xi, \mathbf{q})=\frac{1}{2}\left(\|\xi-\phi\|_{0}^{2}+\|\mathbf{q}-\mathbf{u}\|_{0}^{2}\right) \quad \mapsto \quad \min
$$

subject to

$$
\nabla \phi+\mathbf{u}=0 \quad \text { and } \quad \nabla \cdot \mathbf{q}+\gamma \xi=f
$$

In this problem, the Hodge operator is approximated by the $L^{2}$ projections

$$
\left(*_{0}\right): \mathcal{W}^{0}(\Omega) \mapsto \mathcal{W}^{3}(\Omega) \quad \text { and } \quad\left(*_{1}\right): \mathcal{W}^{1}(\Omega) \mapsto \mathcal{W}^{2}(\Omega)
$$

defined implicitly via the optimization process.

It is possible to solve (4.5)-(4.6) by using Lagrange multipliers to enforce the constraints. However, a better strategy (that also reduces the number of variables) is to note that the constraint equations can be satisfied exactly if the spaces chosen for $\phi_{h}, \mathbf{u}_{h}, \xi_{h}$, and $\mathbf{q}_{h}$ are from a discrete exact sequence. It is also important to note that primal and dual variables can be approximated by discrete exact sequences that are not necessarily defined on the same mesh. Thus, assume that for the primal side we have chosen $\mathcal{W}_{m}^{0}(\Omega)$ and $\mathcal{W}_{m}^{1}(\Omega)$ to approximate $\phi$ and $\mathbf{u}$, respectively, while for the dual side we work with the spaces $\mathcal{W}_{k}^{2}(\Omega)$ and $\mathcal{W}_{k}^{3}(\Omega)$ to approximate $\mathbf{q}$ and $\xi$, respectively. In this manner, each set of variables is represented in the discrete problem by an internal approximation, and we can use the equilibrium equations (rather than the constitutive relations) to eliminate $\xi_{h}$ and $\mathbf{u}_{h}$. This leads to the following discrete minimization problem in terms of $\phi_{h}$ and $\mathbf{q}_{h}$ only:

$$
\min _{\mathcal{W}_{m}^{0}(\Omega) \times \mathcal{W}_{k}^{2}(\Omega)} \frac{1}{2}\left(\left\|\nabla \cdot \mathbf{q}_{h}+\gamma \phi_{h}-f\right\|_{0}^{2}+\left\|\mathbf{q}_{h}+\nabla \phi_{h}\right\|_{0}^{2}\right) .
$$

While this problem appears identical to a least-squares formulation derived directly from (2.5), the manner in which it was obtained retains the information about the origins of the different variables. In particular, we see that in (4.7), the scalar variable is inherited from the primal Dirichlet principle, while the flux is inherited from the dual Kelvin principle. As was shown in section 3.2, when this is taken into account in the choice of approximating finite element spaces, the computational properties of both principles are recovered by (4.7). This is perhaps the most important point of our discussion. Another important distinction between (4.7) and a nodal-based implementation of a least-squares principle is that (4.7) leads to a conservative approximation in the following sense. Once $\phi_{h}$ and $\mathbf{q}_{h}$ are found, we can recover the eliminated dual and primal variables so as to obtain four fields $\phi_{h}, \mathbf{u}_{h}, \xi_{h}$, and $\mathbf{q}_{h}$ that exactly satisfy the relations

$$
\nabla \phi_{h}+\mathbf{u}_{h}=0 \quad \text { and } \quad \nabla \cdot \mathbf{q}_{h}+\gamma \xi_{h}=\Pi_{3} f .
$$

The operator $\Pi_{3}$ that appears above is the $L^{2}$ projection into the subspace $\mathcal{W}_{k}^{3}$ of $L^{2}(\Omega)$, while the discrete Hodge operators can be identified with $L^{2}$ projections from nodal to discontinuous elements and from edge to face elements, respectively. 
5. Conclusions. We have demonstrated that least-squares finite element methods for the first-order Poisson equation can combine the best properties of the classical Dirichlet and Kelvin principles if their implementation uses spaces consistent with the origins of the scalar variable and the flux. In particular, we have shown that a least-squares formulation can be viewed as resulting from a particular choice in the approximation of the Hodge operator. From this point of view, the scalar variable is inherited from the Dirichlet principle and requires approximation by nodal elements. The flux is inherited from the Kelvin principle and must be approximated by $H\left(\Omega\right.$, div) conforming families to enable recovery of optimal $L^{2}$ rates without the addition of curl constraints.

When implemented in this manner, the least-squares finite element method can be deemed superior to both the classical Galerkin and mixed methods because, on the one hand, it provides optimal approximation of all fields with the possibility of recovering an approximation that is conservative in the sense explained earlier, while, on the other hand, it leads to symmetric and positive definite algebraic systems of equations.

Acknowledgment. The authors wish to acknowledge their debt to George Fix, from whom they learned much about mixed and least-squares finite element methods and about science and mathematics in general. Without his guidance and contributions, much of this paper might not have been possible.

\section{REFERENCES}

[1] D. Arnold, Differential complexes and numerical stability, in Proceedings of the International Congress of Mathematicians, Beijing 2002, Li Tatsien, ed., World Scientific, River Edge, NJ, 2002, Vol. 1.

[2] D. N. Arnold, D. Boffi, and R. S. Falk, Quadrilateral H(div) finite elements, SIAM J. Numer. Anal., 42 (2005), pp. 2429-2451.

[3] V. Arnold, Mathematical Methods of Classical Mechanics, Springer, Berlin, 1989.

[4] P. Bochev, A discourse on variational and geometric aspects of stability of discretizations, in Proceedings of the 33th Computational Fluid Dynamics Lecture Series, H. Deconinck, ed., Von Karman Institute of Fluid Dynamics, Lecture Series 2003-05, ISSN0377-8312.

[5] P. B. Bochev And A. C. Robinson, Matching algorithms with physics: Exact sequences of finite element spaces, in Collected Lectures on the Preservation of Stability Under Discretization, D. Estep and S. Tavener, eds., SIAM, Philadelphia, 2002, pp. 145-165.

[6] P. Bochev And C. Scovel, On quadratic invariants and simplectic structure, BIT, 34 (1994), pp. 337-345.

[7] D. Boffi, F. Brezzi, and L. Gastaldi, On the problem of spurious eigenvalues in the approximation of linear elliptic problems in mixed form, Math. Comp., 69 (2000), pp. 121-140.

[8] A. Bossavit, A rationale for "edge-elements" in 3D fields computations, IEEE Trans. Magnetics, 24 (1988), pp. 74-79.

[9] A. Bossavit, Whitney forms: A class of finite elements for three dimensional computations in electromagnetism, IEEE Proceedings, 135 (1988), pp. 493-500.

[10] J. Bramble, R. Lazarov, AND J. Pasciak, A least squares approach based on a discrete minus one inner product for first order systems, Math. Comp., 66 (1997), pp. 935-955.

[11] F. Brezzi And M. Fortin, Mixed and Hybrid Finite Element Methods, Springer, Berlin, 1991.

[12] Z. Cai, R. Lazarov, T. Manteuffel, and S. F. McCormick, First-order system least squares for second-order partial differential equations: Part I, SIAM J. Numer. Anal., 31 (1994), pp. $1785-1799$.

[13] G. Carey and A. Pehlivanov, Error estimates for least-squares mixed finite elements, Math. Model Numer. Anal., 28 (1994), pp. 499-516

[14] C. L. ChAng, Finite element approximation for grad-div type systems in the plane, SIAM J. Numer. Anal., 29 (1992), pp. 452-461.

[15] C. Chang and M. Gunzburger, A finite element method for first order elliptic systems in three dimensions, Appl. Math. Comp., 23 (1987), pp. 171-184. 
[16] G. Fix, M. Gunzburger, And R. Nicolaides, On finite element methods of the least-squares type, Comput. Math. Appl., 5 (1979), pp. 87-98.

[17] G. Fix, M. Gunzburger, And R. Nicolaides, On mixed finite element methods for first-order elliptic systems, Numer. Math., 37 (1981), pp. 29-48.

[18] V. Girault and P.-A. Raviart, Finite Element Methods for Navier-Stokes Equations, Springer, Berlin, 1986.

[19] R. Hiptmair, Canonical construction of finite element spaces, Math. Comp., 68 (1999), pp. $1325-1346$.

[20] R. Hiptmair, Discrete Hodge operators, Numer. Math., 90 (2001), pp. 265-289.

[21] M. Hyman and C. Scovel, Deriving Mimetic Difference Approximations to Differential Operators Using Algebraic Topology, unpublished internal report, Los Alamos National Laboratory, Los Alamos, NM, 1988.

[22] J. Hyman and M. Shashkov, Natural discretizations for the divergence, gradient, and curl on logically rectangular grids, Int. J. Comput Math. Appl., 33 (1997), pp. 88-104.

[23] J. Hyman and M. ShashKov, Adjoint operators for the natural discretizations of the divergence, gradient, and curl on logically rectangular grids, Appl. Numer. Math., 25 (1997), pp. $413-442$.

[24] D. Jesperson, A least-squares decomposition method for solving elliptic equations, Math. Comp., 31 (1977), pp. 873-880.

[25] B. Jiang And L. Povinelli, Optimal least-squares finite element methods for elliptic problems, Comp. Methods Appl. Mech. Engrg., 102 (1993), pp. 199-212.

[26] C. Mattiussi, An analysis of finite volume, finite element and finite difference methods using some concepts from algebraic topology, J. Comput. Phys., 133 (1997), pp. 289-309.

[27] A. I. Pehlivanov, G. F. Carey, and R. D. Lazarov, Least-squares mixed finite elements for second-order elliptic problems, SIAM J. Numer. Anal., 31 (1994), pp. 1368-1377. 